Draft VERSION OCTOBER 7, 2016

Preprint typeset using $\mathrm{IAT}_{\mathrm{E}} \mathrm{X}$ style AASTeX6

With modifications by David W. Hogg and Daniel Foreman-Mackey

\title{
THE POPULATION OF LONG-PERIOD TRANSITING EXOPLANETS
}

\author{
Daniel Foreman-Mackey ${ }^{1,2}$, Timothy D. Morton ${ }^{3}$, David W. HogG ${ }^{4,5,6,7}$, \\ ERIC AGOL ${ }^{2}$, AND BernhaRd SCHÖLKOPF ${ }^{8}$
}

\footnotetext{
${ }^{1}$ danfm@uw.edu; Sagan Fellow

${ }^{2}$ Astronomy Department, University of Washington, Seattle, WA, 98195, USA

${ }^{3}$ Department of Astrophysics, Princeton University, Princeton, NJ, 08544, USA

${ }^{4}$ Simons Center for Data Analysis, 160 Fifth Avenue, 7th floor, New York, NY 10010, USA

${ }^{5}$ Center for Cosmology and Particle Physics, New York University, 4 Washington Place, New York, NY, 10003, USA

${ }^{6}$ Max-Planck-Institut für Astronomie, Königstuhl 17, D-69117 Heidelberg, Germany

${ }^{7}$ Center for Data Science, New York University, 726 Broadway, 7th Floor, New York, NY, 10003, USA

${ }^{8}$ Max Planck Institute for Intelligent Systems Spemannstrasse 38, 72076 Tübingen, Germany
}

\begin{abstract}
The Kepler Mission has discovered thousands of exoplanets and revolutionized our understanding of their population. This large, homogeneous catalog of discoveries has enabled rigorous studies of the occurrence rate of exoplanets and planetary systems as a function of their physical properties. However, transit surveys like Kepler are most sensitive to planets with orbital periods much shorter than the orbital periods of Jupiter and Saturn, the most massive planets in our Solar System. To address this deficiency, we perform a fully automated search for longperiod exoplanets with only one or two transits in the archival Kepler light curves. When applied to the $\sim 40,000$ brightest Sun-like target stars, this search produces 16 long-period exoplanet candidates. Of these candidates, 6 are novel discoveries and 5 are in systems with inner short-period transiting planets. Since our method involves no human intervention, we empirically characterize the detection efficiency of our search. Based on these results, we measure the average occurrence rate of exoplanets smaller than Jupiter with orbital periods in the range 2-25 years to be $2.0 \pm 0.7$ planets per Sun-like star.
\end{abstract}

Keywords: methods: data analysis - methods: statistical — catalogs planetary systems — stars: statistics 


\section{INTRODUCTION}

Data from the Kepler Mission (Borucki et al. 2011) have been used to discover thousands of transiting exoplanets. The systematic nature of these discoveries and careful quantification of survey selection effects, search completeness, and catalog reliability has enabled many diverse studies of the detailed frequency and distribution of exoplanets (for example, Howard et al. 2012; Petigura et al. 2013; Foreman-Mackey et al. 2014; Dressing \& Charbonneau 2015; Burke et al. 2015; Mulders et al. 2015). So far, these results have been limited to relatively short orbital periods because existing transit search methods impose the requirement of the detection of at least three transits within the baseline of the data. For Kepler, with a baseline of about four years, this sets an absolute upper limit of about two years on the range of detectable periods. In the Solar System, Jupiter - with a period of 12 years - dominates the planetary dynamics and, since it would only exhibit at most one transit in the Kepler data, an exo-Jupiter would be missed by most existing transit search procedures.

Before the launch of the Kepler Mission, it was predicted that the nominal mission would discover at least 10 exoplanets with only one or two observed transits (Yee \& Gaudi 2008), yet subsequent searches for these signals have already been more fruitful than expected (Wang et al. 2015; Uehara et al. 2016). However, the systematic study of the population of long-period exoplanets found in the Kepler data to date has been hampered due to the substantial technical challenge of implementing a search, as well as the subtleties involved in interpreting the results. For example, false alarms in the form of uncorrected systematics in the data and background eclipsing binaries can make single-transit detections ambiguous.

Any single transit events discovered in the Kepler light curves are interesting in their own right, but the development of a general and systematic method for the discovery of planets with orbital periods longer than the survey baseline is also crucial for the future of exoplanet research with the transit method. All future transit surveys have shorter observational baselines than the Kepler Mission (K2, Howell et al. 2014: TESS, Ricker et al. 2015; PLATO, Rauer et al. 2014) and given suitable techniques, single transit events will be plentiful and easily discovered. The methodological framework presented in the following pages is a candidate for this task.

A study of the population of long-period transiting planets complements other planet detection and characterization techniques, such as radial velocity (for example Cumming et al. 2008; Knutson et al. 2014; Bryan et al. 2016), microlensing (for example Gould et al. 2010; Cassan et al. 2012; Clanton \& Gaudi 2014; Shvartzvald et al. 2016), direct imaging (for example Bowler 2016), and transmission spectroscopy (for example Dalba et al. 2015). The marriage of the radial velocity and transit techniques is particularly powerful as exoplanets with both mass and radius measurements can be used to study planetary compositions and the formation of planetary systems (for example Weiss \& Marcy 2014; Rogers 2015; Wolfgang et al. 2016). Unfortunately the existing catalog of exoplanets with measured densities is sparsely populated at 
long orbital periods; this makes discoveries with the transit method at long orbital period compelling targets for follow-up observations. Furthermore, even at long orbital periods, the Kepler light curves should be sensitive to planets at the detection limits of the current state-of-the-art radial velocity surveys.

There are two main technical barriers to a systematic search for single transit events. The first is that the transit probability for long-period planets is very low; scaling as $\propto P^{-5 / 3}$ for orbital periods $P$ longer than the baseline of contiguous observations. Therefore, even if long-period planets are intrinsically common, they will be underrepresented in a transiting sample. The second challenge is that there are many signals in the observed light curves caused by stochastic processes - both instrumental and astrophysical - that can masquerade as transits. Even when the most sophisticated methods for removing this variability are used, false signals far outnumber the true transit events in any traditional search.

At the heart of all periodic transit search procedures is a filtering step based on "box least squares" (BLS; Kovács et al. 2002). This step produces a list of candidate transit times that is then vetted to remove the substantial fraction of false signals using some combination of automated heuristics and expert curation. In practice, the fraction of false signals can be substantially reduced by requiring that at least three self-consistent transits be observed (Petigura et al. 2013; Burke et al. 2014; Rowe et al. 2015; Coughlin et al. 2016).

Relaxing the requirement of three transits requires a higher signal-to-noise threshold per transit for validating candidate planets that display only one to two transits. Higher signal-to-noise allows matching the candidate transit to the expected shape of a limbdarkened light curve, as well as ruling out various false alarms. This is analagous to microlensing surveys, for which a planet can only be detected once, thus requiring high signal-to-noise for a reliable detection (Gould et al. 2004).

Recent work has yielded discoveries of long-period transiting planets with only one or two high signal-to-noise transits identified in archival Kepler and K2 light curves by visual inspection (Wang et al. 2013; Kipping et al. 2014b; Wang et al. 2015; Osborn et al. 2016; Kipping et al. 2016; Uehara et al. 2016). These discoveries have already yielded some tantalizing insight into the population of long-period transiting planets but, since these previous results rely on human interaction, it is prohibitively expensive to reliably measure the completeness of these catalogs. As a result, the existing catalogs of long-period transiting planets cannot be used to rigorously constrain the occurrence rate of long-period planets.

In this paper, we develop a systematic method for reliably discovering the transits of large, long-period companions in photometric time series without human intervention. The method is similar in character to the recently published fully automated method used to generate the official DR24 exoplanet candidate catalog from Kepler (Mullally et al. 2016; Coughlin et al. 2016). Since the search methodology is fully automated, we can robustly measure the search completeness - using injection and recovery tests - and use these products to place probabilistic constraints on the occurrence 
rate of long-period planets. We apply this method to a subset of the archival data from the Kepler Mission, present a catalog of exoplanet candidates, and estimate the occurrence rate of long-period exoplanets. We finish by discussing the potential effects of false positives, evaluating the prospects for follow-up, and comparing our results to other studies based on different planet discovery methods.

\section{A FULLY AUTOMATED SEARCH METHOD}

To find long-period exoplanets in the Kepler light curves, we search for individual, high signal-to-noise transit signals using a fully automated procedure that can be broken into three main steps:

1. an initial candidate search using a box-shaped matched filter,

2. light curve-level vetting (using automated model comparison) to remove signals that don't have a convincing transit shape, and

3. pixel-level vetting to remove some astrophysical false positives.

The following sections describe each of these steps in more detail.

The model comparison step (step 2) is the key component of our method that enables robust automation but it is also computationally expensive because we must estimate the marginalized likelihoods of several different models describing a transit and other processes that "look" like transits but are actually caused by noise. This step is conservative: unless a signal is a very convincing transit, it won't pass the test. In practice, this means that all but the highest signal-to-noise events will be rejected at this step. Therefore, in the inexpensive first step - the initial candidate search - we can restrict the candidate list to high signal-to-noise events without a substantial loss in detection efficiency.

\subsection{Step 1 - Initial candidate events}

It is not computationally feasible to run a full model comparison at every conceivable transit time in the light curve so we must first find potentially interesting events. For our purposes, "interesting" means high signal-to-noise and previously unknown.

To generate this list, we use a method much like the standard "box least squares" (BLS; Kovács et al. 2002) procedure with a single (non-periodic) box. After masking

any known transits, we filter the PDC light curves (Stumpe et al. 2012; Smith et al. 2012 ) using a running windowed median with a half-width of 2 days to remove stellar variability. We then compute the signal-to-noise of the depth of a 0.6 day-long top hat on a grid of times spanning the full baseline of observations.

In detail, at each proposal time $t_{0}$, we hypothesize a box-shaped transit with duration $\tau$

$$
m(t)=\left\{\begin{array}{l}
\mu-\delta, \text { if }\left|t-t_{0}\right|<\tau / 2 \\
\mu, \quad \text { otherwise }
\end{array}\right.
$$


Assuming that the uncertainties on the observed fluxes $f(t)$ are Gaussian with known variance $\sigma_{f}^{2}$, the likelihood function for the mean flux $\mu$ and transit depth $\delta$ can be analytically computed to be a two-dimensional Gaussian with mean and covariance given by linear least-squares. This likelihood function provides a natural scalar objective: the signal-to-noise of the measured depth computed as a function of time. In principle this scalar is also a function of duration but we only use a single transit duration because the following steps in this procedure are only sensitive to transits with very high signal-to-noise, and in practice, the final results are insensitive to the specific choice of duration.

To avoid edge effects, we apodize this detection scalar near any large gaps in the time series using a logistic function with width equal to one transit duration. Finally, we estimate the background noise level in the detection scalar time series using a robust running windowed variance estimate of the detection scalar. We accept peaks that are more than 25-times this background noise level as candidates.

For the Kepler light curves, this procedure yields at least one candidate event in about 1 percent of targets. For these targets, we investigate the three highest signal-to-noise events in the following step.

\subsection{Step 2- Light curve vetting}

In this step of the method, the goal is to discard any signals that are not sufficiently "transit-like" in shape. This step is similar to the method independently developed and recently published by the Kepler team (Mullally et al. 2016). To quantify the quality of a candidate, we perform a model comparison between a physical transit model and a set of other parameterized models for systematics. In order for a candidate to pass this vetting step, the transit model must be "preferred" to any other model as measured using the Bayesian Information Criterion (BIC). The BIC is not the optimal choice for this model comparison, but it is more computationally tractable than the alternatives, such as computing thousands of precise marginalized likelihoods or expected utilities for each model. The BIC can be efficiently computed and it exhibits the desired behavior - decreasing with increasing likelihood but flexible models are penalized - and we find that it performs sufficiently well in practice.

For up to three candidate transit times per light curve, we select a contiguous chunk of $P D C$ light curve approximately centered on the proposed transit with no more than 500 cadences (about 10 days) and compute the BIC of each model for this data set. The BIC for a model $k$ in the set of $K$ models is given by

$$
\mathrm{BIC}_{k}=-2 \ln \mathcal{L}^{*}+J \ln N
$$

where the likelihood function $\mathcal{L}$ is evaluated at its maximum, $J$ is the number of free parameters in the model, and $N$ is the number of data points in the data set.

For each model, we describe the data using a Gaussian Process (GP; Rasmussen \& Williams 2006) with a Matérn-3/2 covariance and mean given by the chosen model $m_{k}(t ; \boldsymbol{\theta})$ parameterized by the parameter vector $\boldsymbol{\theta}$. 
We consider the following mean models (this list provides a qualitative justification for each model):

- transit - a limb-darkened, exposure-time integrated transit light curve,

- variability - a pure GP model to capture stellar variability,

- outlier - a single outlier to account for a bad data point,

- step - a step function to describe sudden pixel sensitivity dropouts (SPSDs; for example Christiansen et al. 2013), and

- box - a box to catch signals that are well fit by the search scalar but insufficiently transit-like to be convincing.

The functional forms of these models are given in Appendix $\mathrm{A}$ and the details of the technical methodology of GP fitting are described in Appendix B.

Figure 1 shows representative events that fall into different classes and the corresponding maximum likelihood model. For each candidate event, the BIC of each of these models is computed and the event is only passed as a candidate if the transit model is preferred to all the other models. The box model is the most restrictive comparison, vetoing about half of the candidate events in the Kepler light curves, followed by the variability model. To further restrict to non-grazing transits, we also reject events where the maximum likelihood impact parameter is greater than $1-R_{\mathrm{P}} / R_{\star}$.

Since the search procedure described here was tuned to discover transit signals, we do not consider the distribution or potential astrophysical nature of any models besides the transit model. In the future, it would be interesting to relax this goal and investigate the other model classes; in particular, the box model is sensitive to astrophysical phenomena, notably occultations of white dwarfs. In a cursory investigation it is clear that the majority of signals labeled box in our analysis are noise; however, a subset are likely to be astrophysical in nature.

The reliability of this method of automated vetting is limited by the specific models selected in this step. We find that these are sufficient for the targets discussed below but different target lists or data sets might require additional models to be included for robust selection.

\subsection{Step 3-Pixel-level vetting}

To minimize contamination from background eclipsing binary systems, we require candidate events to pass a centroid shift test similar to the one used in the official Kepler transit search pipeline (Bryson et al. 2013). To measure the centroid shift, we model the flux-weighted centroid traces independently in each coordinate as a multiple of the best-fit transit model and a GP noise model. By properly normalizing the transit model, we measure the in-transit centroid shift $\Delta_{\text {centroid }}$ in pixels. We reject any candidate event where the estimated transit location is more than half a pixel 

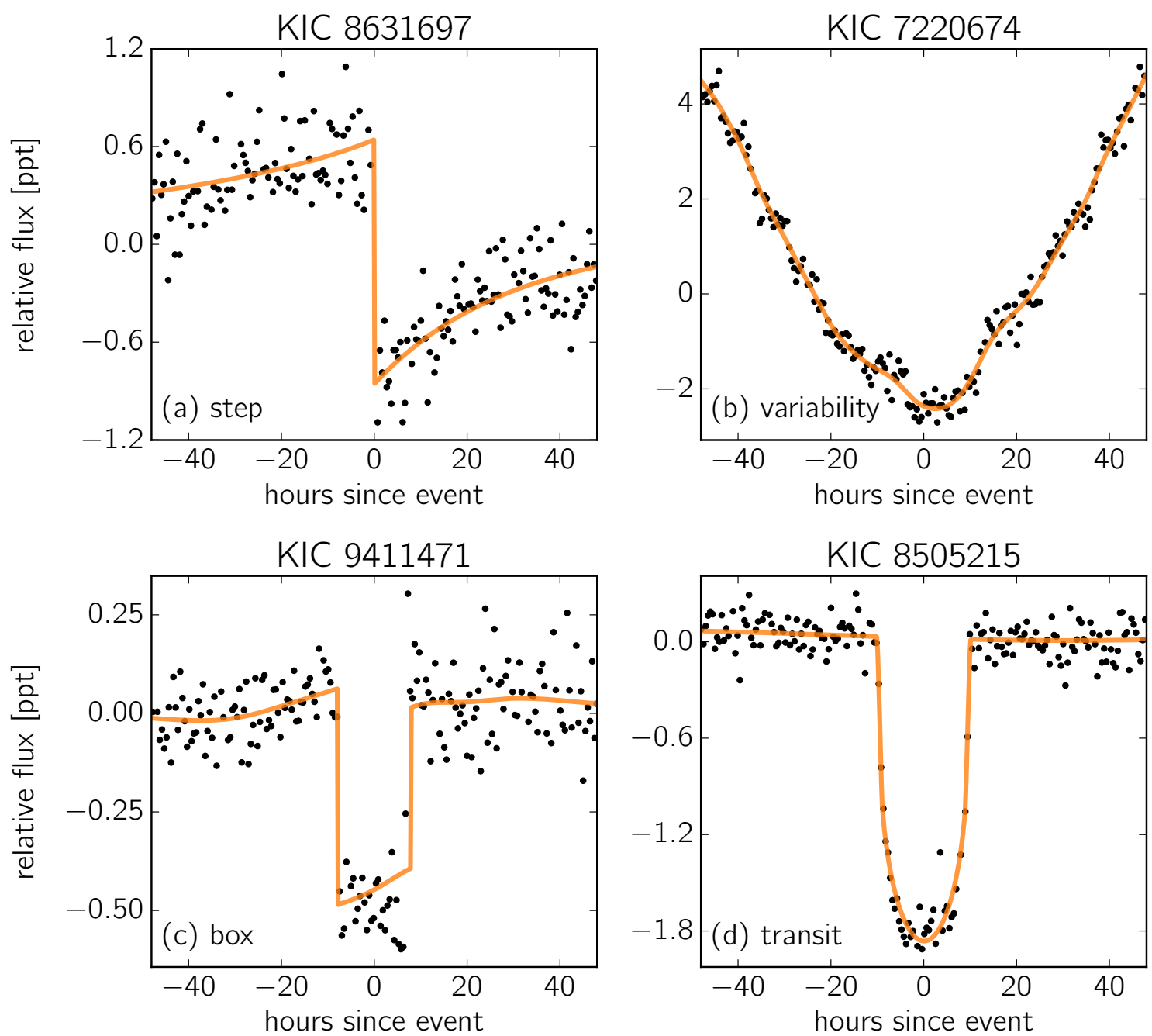

Figure 1. Representative examples of candidate events flagged by the initial search. Each example falls into a different model category and the figure shows the data as black points and the best fit mean model prediction. The examples represent the following model categories: (a) step (b) variability, (c) box, and (d) transit. 
from the out-of-transit centroid

$$
\Delta_{\text {centroid }}\left(\frac{1}{\delta}-1\right)>0.5
$$

where $\delta$ is the observed transit depth (Bryson et al. 2013).

\section{RESULTS: A CATALOG OF LONG-PERIOD TRANSITING EXOPLANET CANDIDATES}

To limit the scope of this paper while still demonstrating the applicability of our method, we search the Kepler archival light curves of the brightest and quietest Sun-like stars for long-period transiting exoplanets. In this section, we describe the target selection process and the parameter estimation procedure.

\subsection{Target selection}

We select the $\sim 40,000$ brightest and quietest $\mathrm{G}$ and $\mathrm{K}$ dwarfs from the Kepler catalog using the most recent catalog of stellar parameters ${ }^{1}$ and the cuts used by Burke et al. (2015):

- $4200 \mathrm{~K} \leq T_{\text {eff }} \leq 6100 \mathrm{~K}$,

- $R_{\star} \leq 1.15 R_{\odot}$,

- data $\operatorname{span} \geq 2$ years,

- duty cycle $\geq 0.6$,

- $K_{p} \leq 15 \mathrm{mag}$, and

- $\mathrm{CDPP}_{7.5 \mathrm{hrs}} \leq 1000$ ppm.

We continue by excluding the light curves of known eclipsing binaries ${ }^{2}$ (Kirk et al. 2016), other known false positives (Coughlin et al. 2016), a planet with known transit timing variations (Kepler-9), and four especially noisy stars (KIC 4482348, KIC 4450472, KIC 5438845, and KIC 10068041). The final catalog contains 39,036 targets and the parameter distribution is shown in Figure 2.

Since these data have already been searched for short-period planets, we assume that all high signal-to-noise candidates with three or more transits have been previously found (Coughlin et al. 2016). To remove these candidates from consideration, we mask the cadences within two transit durations of the time when a short-period planet candidate is known to transit3.

\footnotetext{
1 Parameters from the q1_q17_dr24_stellar table from the NASA Exoplanet Archive Huber et al. 2014, with updates).

${ }^{2}$ http://keplerebs.villanova.edu/

3 We specifically use the q1_q17_dr24_koi from the NASA Exoplanet Archive http:// exoplanetarchive.ipac.caltech.edu/
} 


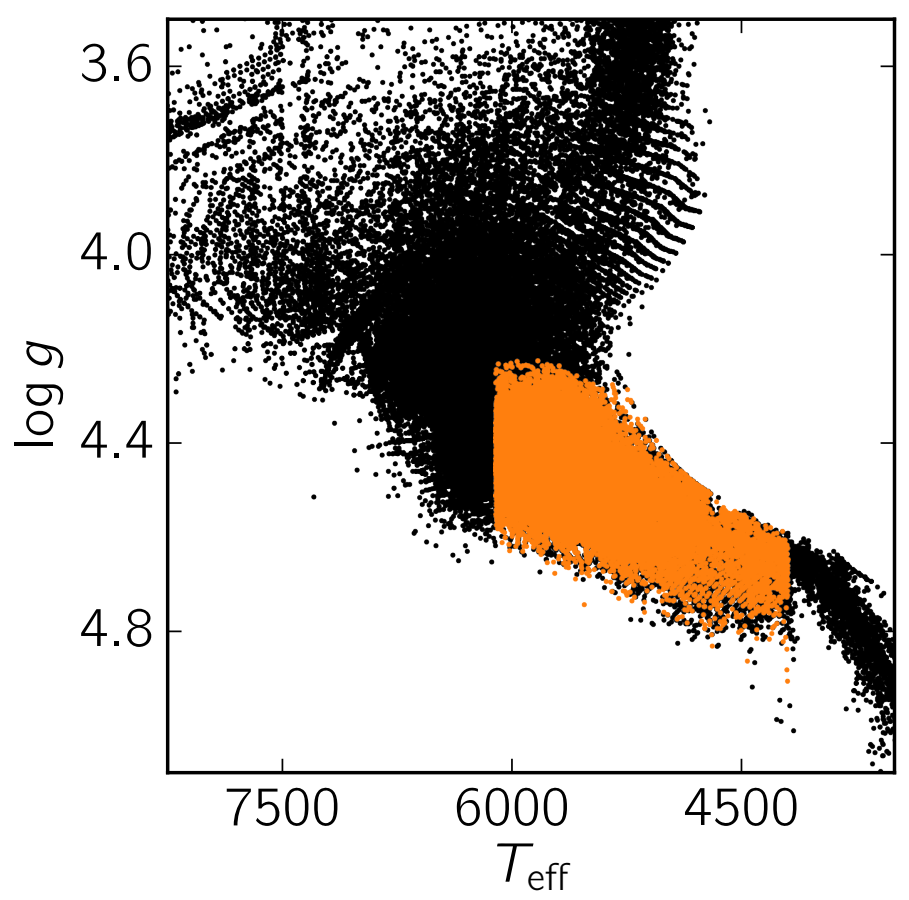

Figure 2. The distribution of stellar parameters for Kepler targets selected for this search (orange) compared to the distribution of the full Kepler target catalog (black).

\subsection{Parameter estimation}

For each transit candidate, we constrain the physical parameters of the system by fitting a section of light curve around each transit using an exposure-time integrated Keplerian orbit with a quadratic limb darkening law for the central body ForemanMackey \& Morton (2016). It has previously been established that the orbital period of a transiting planet with only one transit can still be constrained given a measurement of the stellar density and an assumption about the orbital eccentricity (for example Wang et al. 2015; Osborn et al. 2016). Qualitatively this works because the transit of a bound body cannot have an arbitrary period for a given duration. This is the same argument used to justify the "photoeccentric effect" (Dawson \& Johnson 2012) and the method of "asterodensity profiling" (Kipping et al. 2014a). In particular, this suggests that the periods of single transits in systems with multiple inner planets will be especially well constrained (Kipping et al. 2012). In this paper, we do not take advantage of the extra constraints provided by the inner planets, instead treating each long-period transiting system in isolation, but this would be a good follow-up project.

In the following paragraphs, we describe the components of the probabilistic model used to infer the planet candidates' properties. To perform parameter estimation 
under this model, we use the Markov Chain Monte Carlo (MCMC) package emces $\mathrm{A}^{4}$ (Foreman-Mackey et al. 2013) with an ensemble of 40 walkers. We run each chain until at least 750 independent samples - in most cases, we actually produce thousands of independent samples - are obtained ${ }^{5}$ and discard the first third of the chain as burn-in. The posterior constraints on a few physical parameters for the single transit candidate in the light curve of KIC 8505215 are shown in Figure 3 and all the chains are made available online 6 .

Priors - For each candidate in our sample, we take the constraints on the stellar parameters from the Kepler DR24 stellar properties catalog and assume an empirical beta function prior on the eccentricities based on the observed eccentricity distribution of long-period planets discovered using radial velocities (Kipping 2013a). Table 1 lists all the fit parameters and their prior distributions. Besides these listed priors, we add the extra constraint that no other transits can occur in the baseline of the Kepler observations. This constraint is overly conservative because there is some probability that a second transit could occur in a data gap but we find that, in practice, most of the posterior mass is at longer periods and the period inferences are not significantly affected.

Likelihood function - As above, we model the light curve as a Gaussian Process (GP) with a physical transit model as the mean, and a covariance matrix described by a Matérn-3/2 kernel function. The full likelihood function and some details of GP regression are given in Appendix B. For computational efficiency, we first perform a joint optimization of the physical parameters and GP hyperparameters to find the maximum a posteriori model then keep the hyperparameters fixed and run MCMC sampling for the 11 physical parameters alone.

\section{CATALOG OF TRANSIT CANDIDATES}

Applying the search procedure described in Section 2 to the Kepler light curves of the 39,036 targets selected in Section 3.1, we find 16 convincing transit candidates. Visual inspection of each candidate confirms the reliability of the classification and no candidates are manually removed from the catalog. Of these, three candidates have two transits in the Kepler baseline and the remainder have only one observable transit. The candidates and their inferred physical parameters are listed in Table 2 and the light curves are plotted in Figure 4. The inferred radius and orbital periods of the candidates are compared to the short-period Kepler sample and the Solar System in Figure 5 .

\footnotetext{
4 http://dfm.io/emcee

5 The integrated autocorrelation time is estimated using a robust iterative method as suggested by Alan Sokal: http://www.stat.unc.edu/faculty/cji/Sokal.pdf

6 http://dx.doi.org/10.5281/zenodo.58273
} 


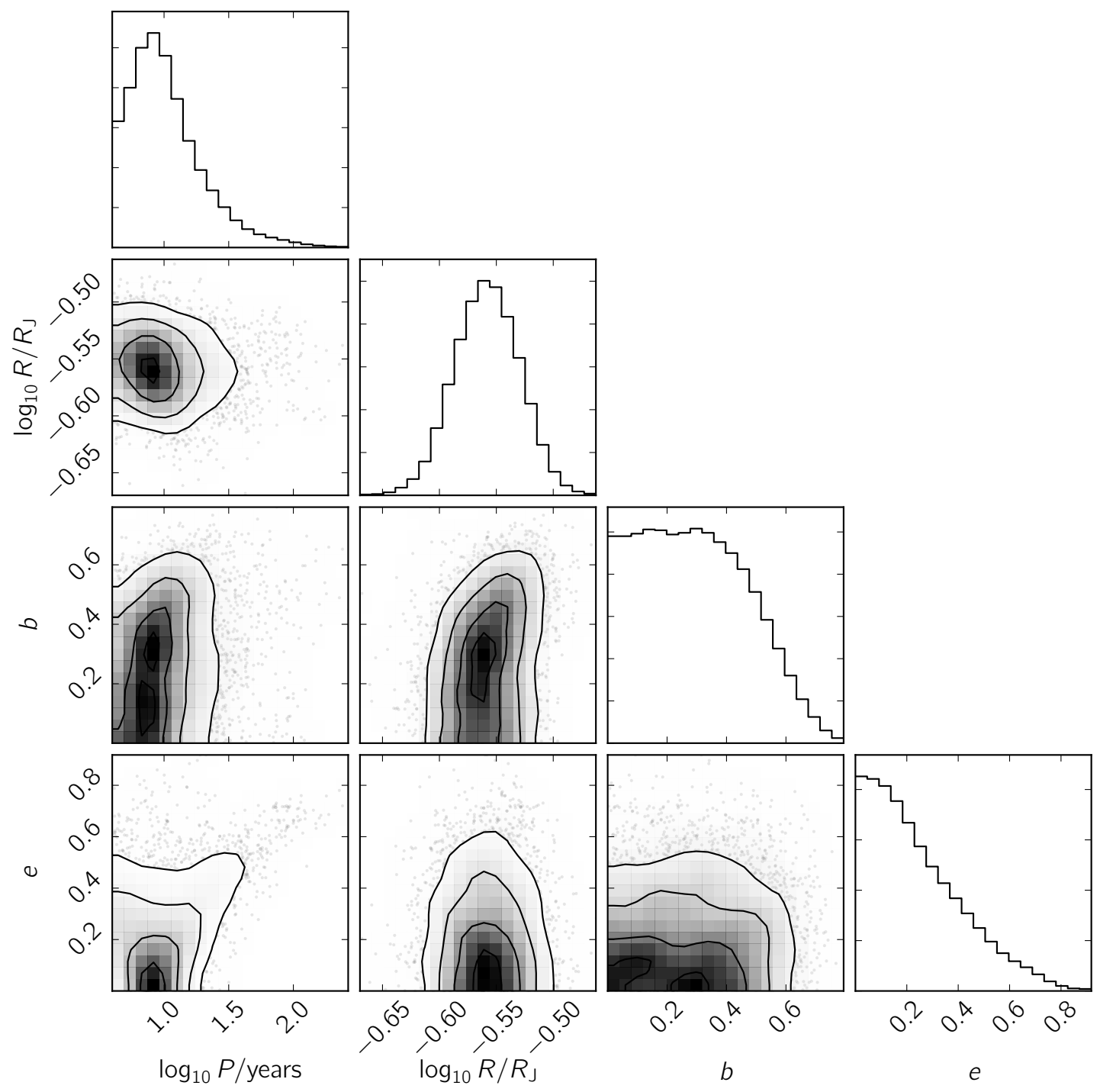

Figure 3. The posterior constraints on the physical parameters for the single transit candidate found in the light curve of KIC 8505215. The contour plots show estimates of the two-dimensional marginalized probability densities and the histograms show the marginalized density for each parameter. This figure was generated using corner.py (Foreman-Mackey 2016).

Two of the shortest period candidates - both with two observed transits - have previously been studied in detail (KIC 8800954 and KIC 3239945; Kipping et al. 2014b, 2016). Table 2 indicates the candidates that were also discovered by earlier searches for long-period transiting systems using visual inspection (Wang et al. 2015; Uehara et al. 2016). The consistency between our results and the earlier catalogs is reassuring. In the light curves of targets with previously known short-period planets, 
Foreman-Mackey, Morton, Hogg, et al.

Table 1. The inferred parameters and priors used in the inference

\begin{tabular}{lccc}
\hline \hline \multicolumn{1}{c}{ name } & symbol & units & prior \\
\hline mean flux & $\log f_{\star}$ & $\ldots$ & $\log f_{\star} \sim \mathcal{U}(-1,1)$ \\
stellar mass & $M_{\star}$ & $M_{\odot}$ & $M_{\star} \sim \mathcal{N}\left(M_{\star, \text { cat }}, \sigma_{M, \star, \text { cat }}\right)$ \\
stellar radius & $R_{\star}$ & $R_{\odot}$ & $R_{\star} \sim \mathcal{N}\left(R_{\star, \text { cat }}, \sigma_{R, \star, \text { cat }}\right)$ \\
& $q_{1}$ & $\ldots$ & $q_{1} \sim \mathcal{U}(0,1)$ \\
limb darkening & $q_{2}$ & $\ldots$ & $q_{2} \sim \mathcal{U}(0,1)$ \\
\hline planet radius & $\log R_{\mathrm{P}}$ & $R_{\odot}$ & $\log R_{\mathrm{P}} \sim \mathcal{U}(-10,2)$ \\
reference time & $t_{0}$ & days & $t_{0} \sim \mathcal{U}\left(t_{\text {cand }}-0.5, t_{\text {cand }}+0.5\right)^{\mathrm{b}}$ \\
semi-major axis & $\sqrt{a} \sin i$ & $R_{\odot}{ }^{1 / 2}$ & $\sqrt{a} \sin i \sim \mathcal{U}\left(-10^{3}, 10^{3}\right) / \sqrt{a}$ \\
$\&$ inclination & $\sqrt{a} \cos i$ & $R_{\odot}{ }^{1 / 2}$ & $\sqrt{a} \cos i \sim \mathcal{U}\left(0,10^{3}\right) / \sqrt{a}$ \\
eccentricity & $\sqrt{e} \sin \omega$ & $\ldots$ & $e \sim \beta(1.12,3.09)^{\mathrm{c}}$ \\
& $\sqrt{e} \cos \omega$ & $\ldots$ & $\omega \sim \mathcal{U}(-\pi, \pi)$
\end{tabular}

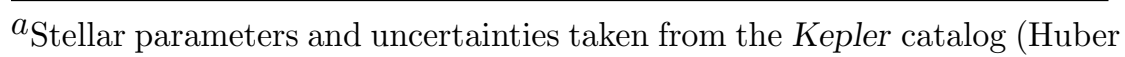
et al. 2014)

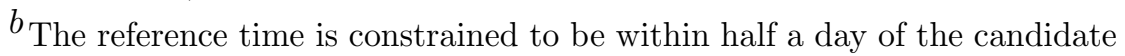
transit time

Kipping 2013b)

Note-There is one further constraint that complicates these priors: the period of the orbit must be longer than some minimum period $P_{\min }$ set by the transit time and the full baseline of Kepler observations.

our automated search did not find any candidates that weren't previously detected by visual inspection (Uehara et al. 2016) and one candidate (KIC 3230491) reported by the human analysis was discarded as grazing by our search. The Planet Hunters citizen science project (Fischer et al. 2012) reported five long-period candidates with one or two observed transits in our target list (Wang et al. 2015). Of these, we also find two (KIC 8410697 and KIC 10842718) although we find a second transit in the KIC 8410697 system that was previously missed. We do not recover the three other candidates reported by Wang et al. (2015): KIC 5536555, KIC 9662267, and KIC 12454613. The transits of these candidates are all low signal-to-noise and they do not pass our initial signal-to-noise threshold. Six of the candidates in Table 2 have not been previously published.

Of the 16 candidates, 5 have known inner planets with three or more observable transits (Coughlin et al. 2016). Given the fact that only 844 of the 39,036 targets had previously known planets, this means that systems with short-period transiting planets are nearly a factor of 20 more likely to host long-period planets accessible by our method than systems with no known inner transiting planets. This difference cannot be accounted for by differences in completeness between targets with known planets 
and without because the average detection efficiency for both populations is consistent within sampling uncertainty. Qualitatively, this suggests that these long-period planets occur with a higher frequency in multi-planet systems or are preferentially aligned with the plane of any inner planets but a more detailed analysis would be needed to make a quantitative statement (see, for example, Tremaine \& Dong 2012; Fang \& Margot 2012; Ballard \& Johnson 2016; Moriarty \& Ballard 2015).

The candidate in the light curve of KIC 4754460 is an individual transit candidate but another deeper eclipse can be found at a Kepler Barycentric Julian Date (KBJD) of 1587.13; right at the beginning of Quarter 17. This eclipse was missed by the automated search because only the second half of the eclipse is observed. The most likely explanation of this system is that the listed candidate is the secondary eclipse of a binary system but we will keep the candidate in the list and treat this effect statistically in Section 7 .

Five candidate transit events in the light curves of four targets were rejected because of a significant centroid shift or a large impact parameter. These events are probably astrophysical eclipses from binary star systems that were not found by previous studies of long-period eclipsing binary systems. We do not consider these events further in the following analysis but Table 3 lists these events and their properties for posterity.

\section{EMPIRICAL SEARCH COMPLETENESS}

To measure the completeness of the search procedure described in Section 2, we exploit the fact that transit signals are sparse and rare. Therefore, most light curves contain no transits and we can reliably measure the recovery rate of our method on synthetic transit signals - with known properties - injected into real light curves. This procedure is standard practice in the transit literature and it has been used to determine the completeness of the KOI catalog (Christiansen et al. 2013, 2015) and other independent transit searches (Petigura et al. 2013; Dressing \& Charbonneau 2015; Foreman-Mackey et al. 2015).

To reliably capture the full structure of the search completeness function, the simulations must sample the (high-dimensional) space of all properties that affect the probability of detecting a transit: the stellar properties (including variability amplitudes and time scales), the planet's physical properties and orbital elements, and any observational effects (noise, spacecraft pointing variations, etc.). For the modest goals of this paper, we only need a robust constraint on the transit detection efficiency integrated across the target sample but, even so, many simulations per star are required.

The procedure for measuring the recovery rate of simulated transits is as follows:

1. First, a star is randomly selected from the target list, and the $P D C$ light curve and stellar properties for that star are loaded. 


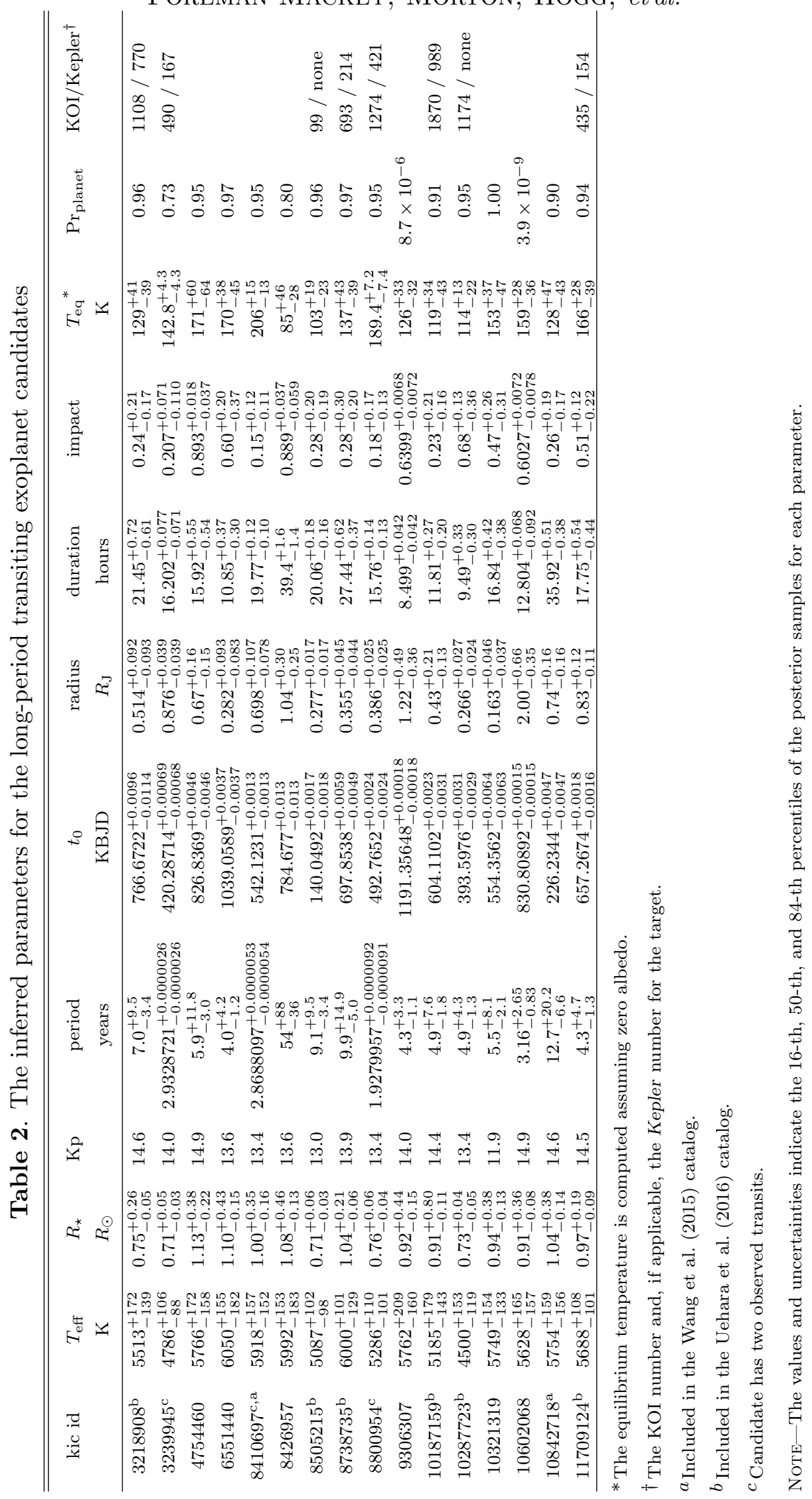


THE POPULATION OF LONG-PERIOD TRANSITING EXOPLANETS

Table 3. The signals rejected with a centroid shift or large impact parameter

\begin{tabular}{ccccc}
\hline \hline \multirow{2}{*}{ kic id } & $\begin{array}{c}\text { time } \\
\text { KBJD }\end{array}$ & $\begin{array}{c}\text { depth } \\
\text { ppt }\end{array}$ & $\begin{array}{c}\text { duration } \\
\text { hours }\end{array}$ & reason \\
\hline 3230491 & 315.3 & 9.0 & 7.4 & impact \\
6342758 & 553.9 & 10.3 & 9.9 & impact \\
8463272 & 641.0 & 35.5 & 4.8 & impact \\
8463272 & 1206.7 & 35.5 & 4.8 & impact \\
10668646 & 1449.2 & 5.7 & 12.4 & centroid \\
\hline
\end{tabular}

2. Planetary properties are sampled from the distributions listed in Table 4 with phase uniformly distributed across the baseline of observations. These properties are re-sampled until the transit is visible in at least one non-flagged cadence.

3. The transit signal induced by this planet is computed and multiplied into the $P D C$ light curve.

4. The transit search method described in Section 2-including de-trending and all automated vetting - is applied to this light curve with the injected transit signal.

5. This candidate is flagged as recovered if at least one transit within one transit duration passes all the cuts imposed by the automated vetting.

The fraction of recovered simulations as a function of the relevant parameters gives an estimate of the probability of detecting an exoplanet transit with a given set of parameters, conditioned on the fact that it transits the star during a time when the star was being observed by Kepler. We will call this function $Q_{\operatorname{det}, k}(\boldsymbol{w})$ where $\boldsymbol{w}$ is the set of all parameters affecting the transit detectability and $k$ is an index running over target stars.

Figure 6 shows the fraction of recovered simulations as a function of planet radius and orbital period based on 819,752 injected signals. This figure shows the transit detection efficiency falling with decreasing planet radius. This is the expected behavior because the depth (and signal strength) of a transit scales with the area ratio between the planet and the star. There is also a slight decrease in the completeness to larger planet radius. This trend is introduced in steps 2 and 3 of the search procedure where the tuning parameters were chosen to maximize the yield of convincing small transit discoveries. The decreasing completeness with orbital period is less intuitive because, on average, the signal strength should increase as the duration of the transit increases. In this case, this simplistic treatment misses two important factors. First, in step 1 of the search procedure (Section 2.1) only a single transit duration is used and second, longer transits are less easily distinguished from stellar variability and they will, therefore, be discarded in the conservative light curve vetting step (Section 2.2). 

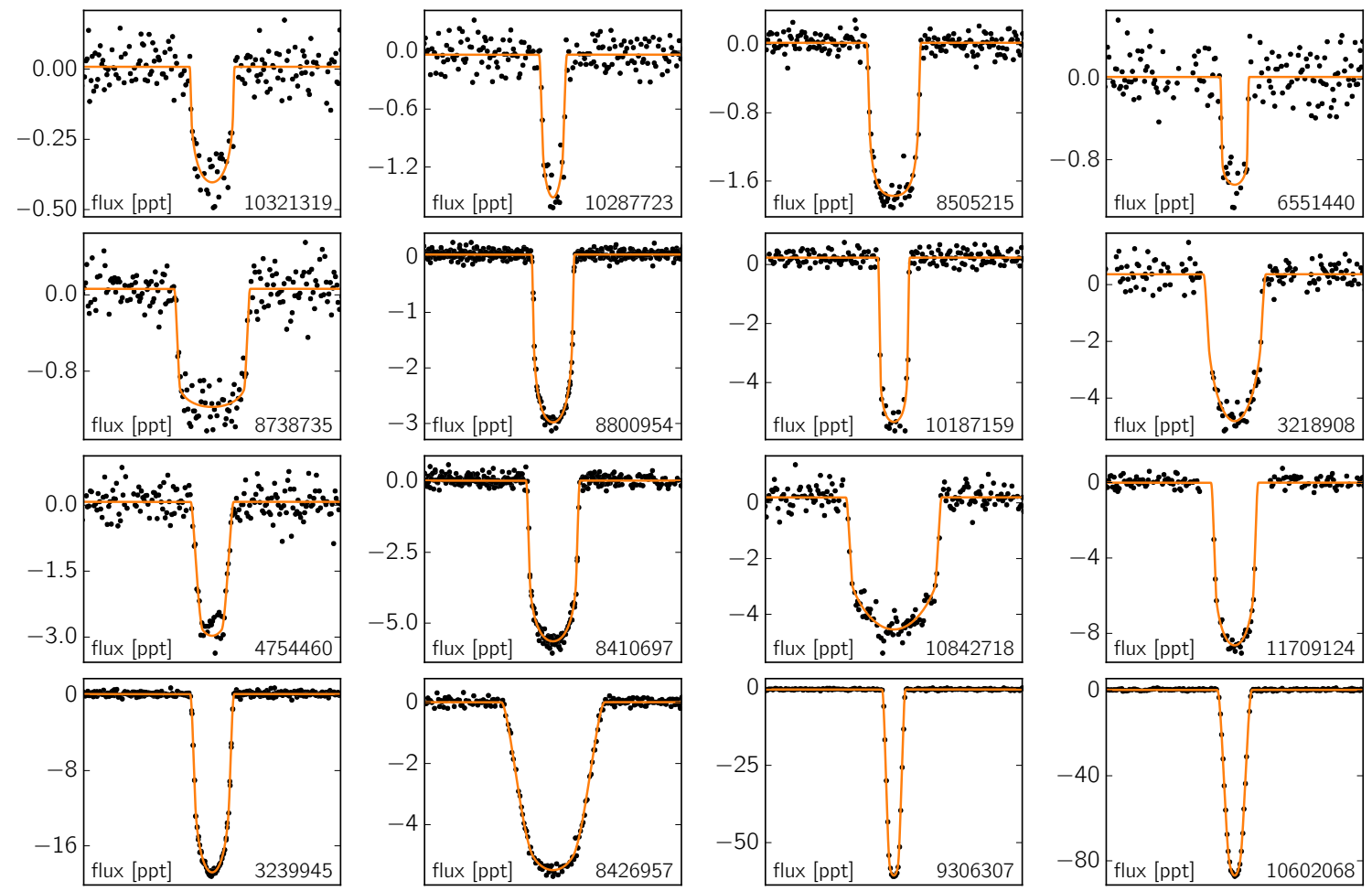

Figure 4. Sections of $P D C$ light curve centered on each candidate (black) with the posteriormedian transit model over-plotted (orange). The y-axis shows the relative apparent flux of the light curve in parts per thousand (ppt). Candidates with two transits are folded on the posterior-median period. The plots are ordered by increasing planetary radius from the top-left to the bottom-right. 


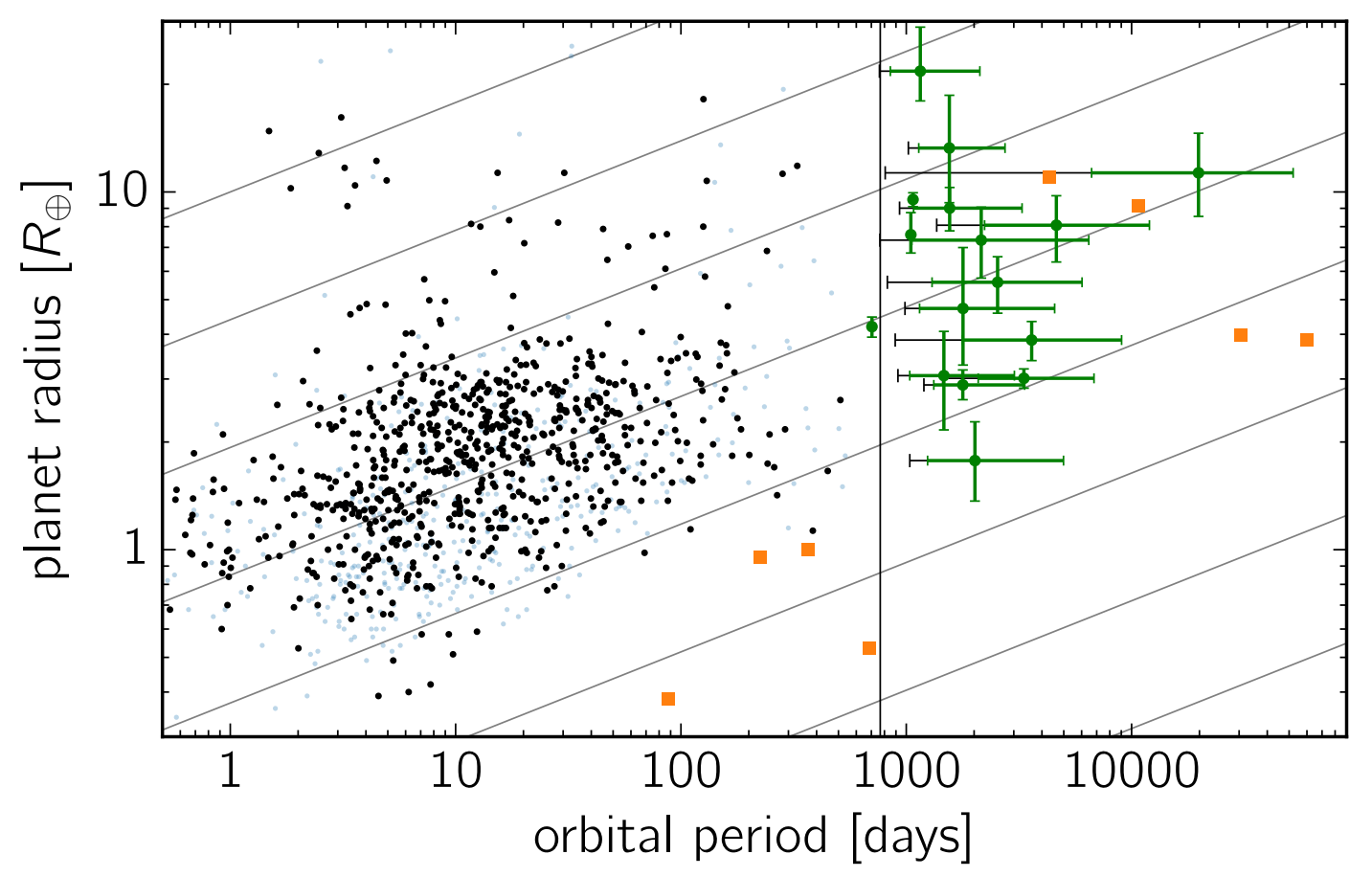

Figure 5. The catalog of long-period transiting exoplanet candidates (green points with error bars) compared to the Kepler candidates (blue points) and confirmed planets (black points; Morton et al. 2016) found in our target sample, and the Solar System (orange squares). The thin black error bars to the left of each candidate indicate the minimum period allowed for each candidate by the prior assumption that no other transit occurred during the baseline of Kepler observations of the target. The vertical solid line shows the absolute maximum period accessible to transit searches that require at least three transits in the Kepler data.

This detection efficiency must then be combined with the geometric transit probability function and the window function. For the star $k$, the geometric transit probability is given by (Winn 2010)

$$
\begin{aligned}
Q_{\text {geom }, k}(\boldsymbol{w}) & =\frac{R_{\star, k}+R}{a_{k}} \frac{1+e \sin \omega}{1-e^{2}} \\
& =\left[\frac{4 \pi^{2}}{G M_{\star, k}}\right]^{1 / 3}\left[\frac{1+e \sin \omega}{1-e^{2}}\right]\left(R_{\star, k}+R\right) P^{-2 / 3}
\end{aligned}
$$

where $R$ is the planet radius, $P$ is the orbital period, $e$ is the orbital eccentricity, $\omega$ is the argument of periastron, $R_{\star, k}$ is the radius of star $k$, and $M_{\star, k}$ is the star's mass. All of these parameters are included in $\boldsymbol{w}$.

In Equation (4), the term $\left(R_{\star, k}+R\right)$ takes grazing transits into account. This might seem counter intuitive because, as part of the search procedure, we rejected candidates where the maximum likelihood model had a grazing transit. However, since the measurement of $Q_{\mathrm{det}, k}$ included a cut on the measured impact parameter, the 
$Q_{\text {det }, k}$ term already takes this effect into account. In other words, $Q_{\text {det }, k}$ quantifies the probability that a transit of a given shape will be detected given that it transits at all and $Q_{\text {geom }, k}$ - the way it is written in Equation (4) - is the marginalized probability that the system will transit given its physical parameters.

Approximating the window function using a binomial probability of observing at least one transit, we find (following Burke \& McCullough 2014)

$$
Q_{\text {win }, k}(\boldsymbol{w})= \begin{cases}1-\left(1-f_{\text {duty }, k}\right)^{T_{k} / P} & \text { if } P \leq T_{k} \\ T_{k} f_{\text {duty }, k} / P & \text { otherwise }\end{cases}
$$

where $f_{\text {duty }, k}$ is the duty cycle and $T_{k}$ is the full observation baseline for target $k$.

Combining these detection effects, the total detection efficiency is given by

$$
Q_{k}(\boldsymbol{w})=Q_{\operatorname{det}, k}(\boldsymbol{w}) Q_{\operatorname{win}, k}(\boldsymbol{w}) Q_{\text {geom }, k}(\boldsymbol{w}) .
$$

So that our planet candidate catalog can be easily used for other projects, we also provide an analytic approximation to the relevant integrated detection efficiency function

$$
Q_{\operatorname{det}}(P, R)=\sum_{k=1}^{K} \int Q_{\operatorname{det}, \mathrm{k}}(\boldsymbol{w}) p\left(\boldsymbol{w}_{\{P, R\}}\right) \mathrm{d} \boldsymbol{w}_{\{P, R\}}
$$

where $p\left(\boldsymbol{w}_{\{P, R\}}\right)$ is the prior distribution of all the parameters except the period and radius. We find that a good fit to this integrated completeness is given by the function

$$
Q_{\text {det }}(P, R) \approx \frac{\min [\max [a(P) b(R), 0], 1]}{1+\exp \left[-k(P)\left(\ln R / R_{\mathrm{J}}-x(P)\right)\right]}
$$

where

$$
\begin{gathered}
a(P)=a_{1} \ln P / \mathrm{yr}+a_{2}, \quad b(R)=b_{1} \ln R / R_{\mathrm{J}}+b_{2}, \\
k(P)=k_{1} \ln P / \mathrm{yr}+k_{2}, \text { and } \quad x(P)=x_{1} \ln P / \mathrm{yr}+x_{2} .
\end{gathered}
$$

When fit to the set of 819,752 injected transits, the best fit parameters are given in Table 5 and the approximation is plotted in Figure 7. Note that we do not use this approximation in the following analysis but instead compute the relevant integrals using the injection results directly.

\section{THE OCCURRENCE RATE OF LONG-PERIOD EXOPLANETS}

Using the catalog of exoplanet discoveries (Section 4) and the measurement of the search completeness (Section 5), we can now estimate the occurrence rate of long-period exoplanets. To simplify the analysis, we will make the strong assumption that none of the candidates are astrophysical false positives (the eclipse or occultation of a stellar mass companion, around either the target star or a faint background star). We revisit this assumption and discuss its validity in the following section. As a further simplification, we also neglect the measurement uncertainties on the planet 
The POPUlATION OF LONG-PERIOD TRANSITING EXOPLANETS

Table 4. Distributions of physical parameters for transit simulations

\begin{tabular}{|c|c|}
\hline name & distribution \\
\hline period & $\log P \sim \mathcal{U}(\log 2 \mathrm{yr}, \log 25 \mathrm{yr})$ \\
\hline radius ratio & $\log R_{\mathrm{P}} / R_{\star} \sim \mathcal{U}(\log 0.02, \log 0.2)$ \\
\hline impact parameter & $b \sim \mathcal{U}\left(0,1+R_{\mathrm{P}} / R_{\star}\right)$ \\
\hline \multirow{2}{*}{ eccentricity } & $e \sim \beta(1.12,3.09)^{\mathrm{a}}$ \\
\hline & $\omega \sim \mathcal{U}(-\pi, \pi)$ \\
\hline \multirow{2}{*}{ limb darkening } & $q_{1} \sim \mathcal{U}(0,1)$ \\
\hline & $q_{2} \sim \mathcal{U}(0,1)$ \\
\hline
\end{tabular}

Kipping (2013b)

Table 5. The fit parameters for the analytic approximation to the completeness function

\begin{tabular}{cc|cc}
\hline \hline & & & \\
parameter & \multicolumn{1}{c}{ value } & parameter & value \\
\hline$a_{1}$ & -0.13 & $k_{1}$ & 0.70 \\
$a_{2}$ & 0.95 & $k_{2}$ & 3.06 \\
$b_{1}$ & -0.20 & $x_{1}$ & -0.07 \\
$b_{2}$ & 0.90 & $x_{2}$ & -0.91 \\
\hline
\end{tabular}

parameters (including orbital period). This assumption is justified because we are only making high-level measurements of the mean occurrence rate in bins larger than the uncertainties.

Assuming a Poisson likelihood, the occurrence rate density in a volume $V$ - defined as $P_{\min } \leq P<P_{\max }$ and $R_{\min } \leq R<R_{\max }$ - is (see, for example, the Appendix of Foreman-Mackey et al. 2014)

$$
\Gamma_{V} \equiv \frac{\mathrm{d}^{2} N}{\mathrm{~d} \ln P \mathrm{~d} \ln R}=\frac{C\left(P_{\min }, P_{\max } ; R_{\min }, R_{\max }\right)}{Z\left(P_{\min }, P_{\max } ; R_{\min }, R_{\max }\right)}
$$

where $N$ is the expected number of planets per $\mathrm{G} / \mathrm{K}$ dwarf, $C(\cdots)$ is the number of detected planets in the volume, and

$$
Z\left(P_{\min }, P_{\max } ; R_{\min }, R_{\max }\right)=\sum_{k=1}^{K} \int p\left(\boldsymbol{w}_{\{P, R\}}\right) Q_{k}(\boldsymbol{w}) \mathbf{1}[P, R \in V] \mathrm{d} \boldsymbol{w}
$$

where $p\left(\boldsymbol{w}_{\{P, R\}}\right)$ is the prior distribution of all the parameters except the period and radius and $\mathbf{1}[\cdot]$ is 1 if the argument is satisfied and 0 otherwise. Using the $J$ injections 


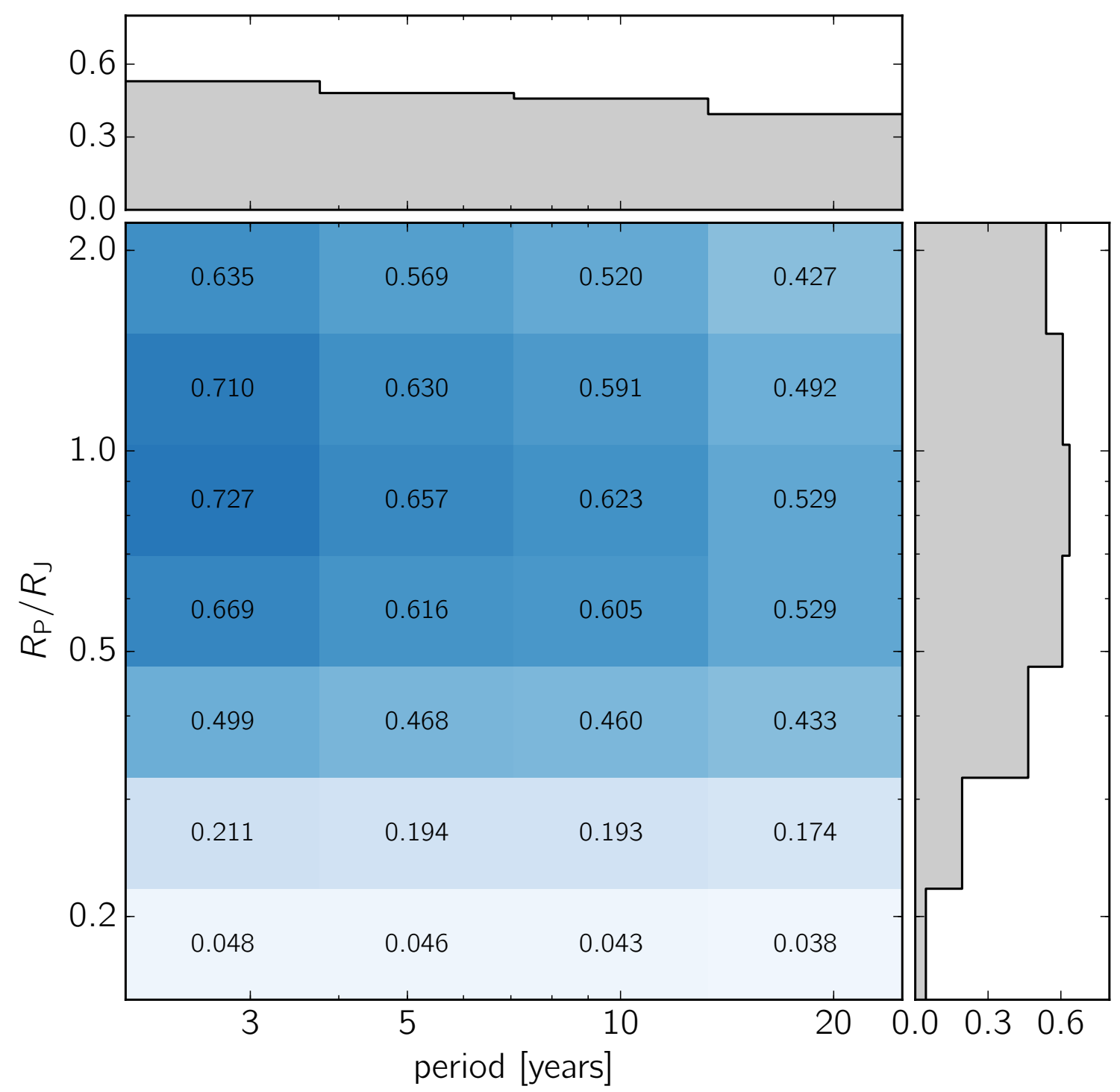

Figure 6. An empirical estimate of the search completeness as a function of planet radius and orbital period. In each bin, the completeness is estimated by the fraction of recovered simulations. The projected histograms show the integrated completeness as independent functions of period and radius. 


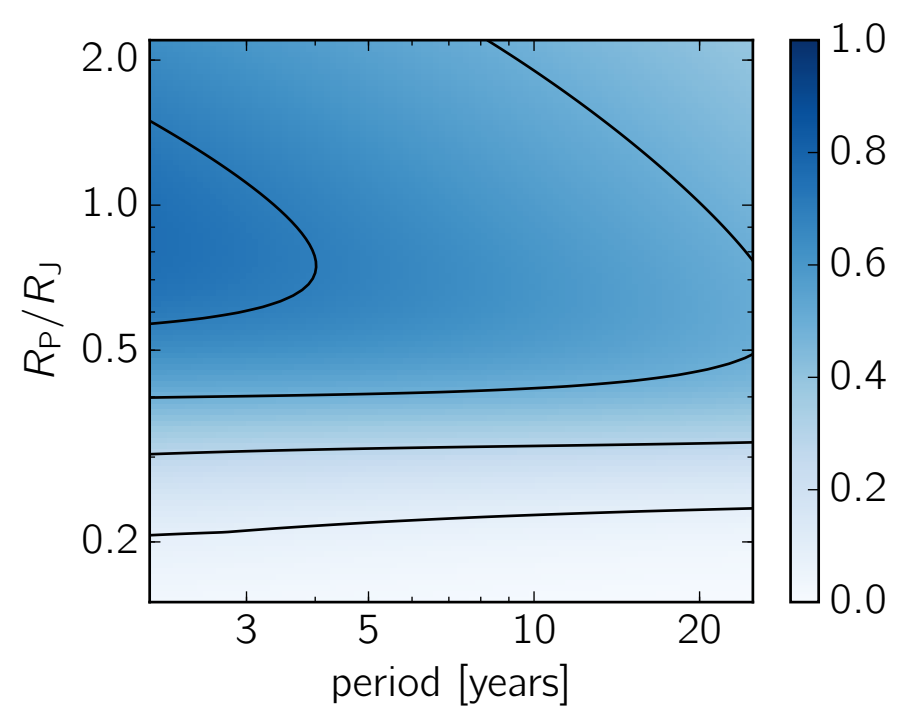

Figure 7. An analytic approximation to Figure 6 with the same color scale. The contours indicate the $0.1,0.3,0.5$, and 0.7 levels.

sampled uniformly in period and radius and other parameters from $p\left(w_{\{P, R\}}\right)$,

$$
Z\left(P_{\min }, P_{\max } ; R_{\min }, R_{\max }\right) \approx \frac{K V}{J} \sum_{j=1}^{J} Q_{k_{j}}\left(w^{(j)}\right)
$$

where the sum is over all injections in the volume $V$.

Using the injection results from Section 5 and the catalog of discoveries from Section 4, we compute the occurrence rate in the period range 2 to 25 years and in two radius bins between 0.1 and $1.0 R_{\mathrm{J}}$. The calculated occurrence rates are listed in Table 6. Integrating the two bin model in this range, we find an expected occurrence rate of

$$
N_{0.1 R_{\mathrm{J}}-1 R_{\mathrm{J}}, 2 \mathrm{yr}-25 \mathrm{yr}}=2.00 \pm 0.72 \text { planets }
$$

per $\mathrm{G} / \mathrm{K}$ dwarf with radii in the range $0.1 R_{\mathrm{J}}-1 R_{\mathrm{J}}$ and periods in the range $2 \mathrm{yr}-25 \mathrm{yr}$. This result is qualitatively consistent with the Solar System where there is one planet Jupiter - in this parameter range and Saturn is just outside the range with an orbital period of 29 years. In Section 8, we compare with similar occurrence rate estimates from the literature.

The occurrence rates given here should be interpreted with a few caveats in mind. First, when we inferred the periods of the planets with only one transit, we assumed that the period was long enough that no other transit occurred during the Kepler lifetime. This neglects the small but non-negligible posterior probability - less than one percent for the typical candidate - that a second transit might have occurred in a data gap. All of the candidates in our catalog are consistent with having periods this long but the geometric transit probability decreases quickly with orbital period. For the 
Table 6. The occurrence rate density in two radius bins

\begin{tabular}{cccc}
\hline \hline$R_{\min }\left[R_{\mathrm{J}}\right]$ & $R_{\max }\left[R_{\mathrm{J}}\right]$ & rate density $^{\mathrm{a}}$ & integrated rate $^{\mathrm{b}}$ \\
\hline 0.1 & 0.4 & $0.45 \pm 0.20(0.36 \pm 0.16)$ & $1.57 \pm 0.70(1.26 \pm 0.56)$ \\
0.4 & 1.0 & $0.18 \pm 0.07(0.16 \pm 0.06)$ & $0.42 \pm 0.16(0.36 \pm 0.14)$ \\
0.1 & 1.0 & $0.24 \pm 0.07(0.22 \pm 0.06)$ & $1.41 \pm 0.41(1.29 \pm 0.37)$ \\
\hline
\end{tabular}

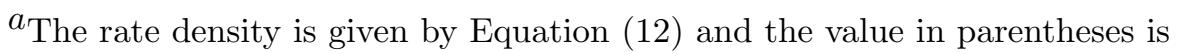
computed assuming one candidate is a false positive (Equation 17 ).

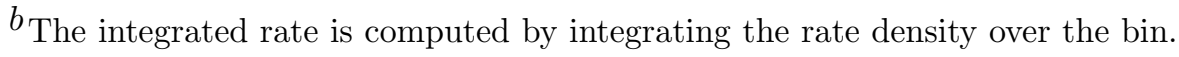
Note that the first two rows do not sum to the last row because each row is computed assuming that the rate density is uniform across the bin.

Note-These values are computed in the period range 2-25 years.

purposes of this paper, we neglect this effect because its rigorous treatment is subtle, but comment that this would only ever decrease the occurrence rate estimate. Second, we assume that each planet candidate transits the star that is characterized by Huber et al. (2014); we assert that each planet does not transit a fainter companion star or a background star. If the planet does transit a companion star, then the companion star must be fainter, and hence denser, causing the period to be underestimated. If the planet transits a background star, it is more likely to be a giant star due to Malmquist bias, hence the density of the star and period of the planet would be overestimated. Either of these scenarios has a small probability, so we expect that our population estimates will stand, while the parameter estimates for individual candidates should be taken as provisional until more detailed follow-up is carried out, including high-contrast imaging, high-resolution spectroscopy, and parallax measurements. Third, we assume that the Huber et al. (2014) parameters are accurate for each star that is transited by a planet candidate, and that each transit is unaffected by blending. Malmquist bias, Eddington bias, and metallicity bias may affect the stellar parameters (Gaidos \& Mann 2012), and so we again caution that the individual parameter estimates should be taken as provisional until more detailed follow-up is completed.

\section{ASTROPHYSICAL FALSE POSITIVES}

Various configurations of eclipsing binary stars can mimic the signal of a transiting planet. However, the occurrence rate calculation presented in the preceding section assumes no astrophysical false positives among the candidates identified in this work. In this Section we explore the validity of this assumption.

While an eclipsing binary (EB) typically produces a photometric dip much deeper than a transiting planet, the depth of the signal may be comparable to that of a planet if the eclipse is grazing, or if the EB only comprises a small fraction of the total light in the photometric aperture - a so-called blended eclipsing binary (BEB). Additionally, if a binary star has an eccentric orbit, it may be oriented so as to present only a 
secondary occultation and not a primary eclipse, causing a shallow and potentially flat-bottomed photometric dip without an accompanying tell-tale deep primary signal.

To determine to what extent the catalog of detections presented in this work may contain such false positives, we simulate populations of detected signals to predict how many we should expect. To accomplish this, we use the Python package exosyspop (Morton \& Foreman-Mackey 2016), which we developed for this purpose and utilizes the isochrones, vespa, and batman packages (Morton 2015a b; Kreidberg 2015) for simulations of stellar populations and their eclipses.

With exosyspop one can define the parameters of a population model and generate synthetic catalogs according to the model (and the parameters of a survey) very efficiently. For example, a population of EBs may be defined by a binary fraction, power-law distributions in mass ratio and period (within given bounds), and a beta distribution for eccentricity. This population, initialized with a catalog of target stars (each of which has a duty cycle and total span of observation), may then be "observed," returning a catalog of objects detectable via either primary or secondary eclipse (according to randomly oriented orbital geometries and accounting for observation duty cycle and data span). This synthetic catalog includes signal-to-noise estimates of both the primary and secondary eclipses, the number of detected primary and secondary eclipses, and the trapezoidal shape parameters of each detection (depth, duration, and ingress-to-duration ratio, as defined in Morton 2012).

In order to predict how many EBs or BEBs we might expect to detect in this particular search of Kepler data, we first need to choose reasonable parameters for the binary star population. To do this, we calibrate the population parameters using the catalog of detected Kepler eclipsing binaries. We find that a binary fraction of $25 \%$ between periods of 20 days and 25 years with a log-flat period distribution and eccentrities distributed according to $\beta(0.8,2.0)$ is able to reproduce well both the number and period distribution of observed Kepler EBs between 20 and 1000 days. We thus fix these binary star population parameters for our subsequent EB and BEB simulations.

To simulate synthetic populations of EB detections, we assign binary stars to the Kepler target list described in Section 3.1 according to the above EB population parameters. We consider an EB to be detected if it presents fewer than three eclipses (either primary or secondary, but not both), if the signal-to-noise ratio is $>15$, and the duration of the detected eclipse is $<2.5 \mathrm{~d}$. In 100 realizations of these synthetic observations, we see $7.2 \pm 2.5$ single- or double-eclipsing EB signals.

To simulate BEBs, we assume an exponentially varying background field star density across the Kepler field, from $0.005 \operatorname{arcsec}^{-2}$ at a Galactic latitude $b=20$ to $0.05 \operatorname{arcsec}^{-2}$ at $b=5$ (matching up well with the simulations of Morton \& Johnson (2011) at many different Galactic latitudes). Each Kepler target star is then assigned a number of background stars drawn from a Poisson distribution with mean given by the expected number of stars to be found within a circle of 4 arcsec radius, given the appropriate density at its Galactic lattitude. We draw the specific background 
stars from a TRILEGAL (Girardi et al. 2005) field star simulation toward the center of the Kepler field. Binary companions are then assigned to these background stars according to the same stellar binary population distribution as the EB population above, and synthetic detected populations are "observed" according to the same rules (accounting appropriately for the diluted eclipse depths in the Kepler bandpass). In 100 synthetic observations, we see an average of only 0.41 detected BEBs.

These prediction results suggest that we should indeed expect to see some astrophysical false positives in our search. However, this does not mean that we should fear that $\sim 7$ of the planet candidates might be EBs. In particular, we note that these simulations do not include the full vetting procedure described in Section 4 , and it is likely that the three impact-parameter-rejected candidates are EBs and that the centroid-rejected candidate is a BEB. Thus, we might expect maybe two or three additional false positives among our planet candidates.

In order to more precisely quantify which of the candidates might indeed be false positives, we can inspect the synthetic observation simulations in more detail. In particular, we can analyze the shape distribution of the different scenarios and compare them with the observed shapes of the actual Kepler detections in order to quantify the probability that each of them may be a false positive. These distributions and the observed shape parameters are plotted in Figure 8.

Following the method of Morton (2012) used to compute false positive probabilities for the regular Kepler KOI catalogs (Morton et al.|2016), we can calculate the posterior probability for each of our candidates to belong to each of the three scenarios we consider (EB, BEB, or planet) as follows:

$$
\operatorname{Pr}_{i}=\frac{\pi_{i} \mathcal{L}_{i}}{\sum_{j} \pi_{j} \mathcal{L}_{j}},
$$

where the $\pi$ factors are the hypothesis priors, $\mathcal{L}$ are the hypothesis likelihoods, and the sum over $j$ is over all the hypotheses. In this case, we determine the relative hypothesis priors from the synthetic observations, using the mean numbers of "observed" EBs (7.2) and BEBs (0.4), and choosing the expected number of planets to be 12 . We calculate the hypothesis likelihoods using the depth/duration distributions of synthetic populations of each scenario and evaluating these distributions at the observed depths and durations of each candidate signal. To estimate the expected shape distribution of the planet scenario, we define a custom exosyspop population of planets according to the two-bin population model described by the median posterior values in Table 6 and generate a population of 1000 detected signals.

We list the probability that each candidate is a planet in Table 2. We find that the two deepest signals in our candidate catalog (9306307 and 10602068) are very likely to be EBs, though we note that this result may be dominated by the fact that our planet population is fixed to have a maximum radius of $1 R_{\mathrm{J}}$. Most of the rest of the candidates have false positive probabilities below $10 \%$. We do note that as discussed in Section 4, 4754460 (for which we calculate a $5 \%$ false positive probability) does 
show a partial deep eclipse right at the end of the Kepler data that indicates that it is most likely an EB. Apart from this, the expected number of false positives among the candidates with $R<R_{\mathrm{J}}$, according to these calculations, is about one.

In light of this result, we demonstrate the sensitivity of our measured occurrence rates on contamination by computing a second constraint on $\Gamma_{V}$ for each volume with one candidate removed. In this case, Equation (12) would be replaced by

$$
\tilde{\Gamma}_{V} \equiv \frac{C\left(P_{\min }, P_{\max } ; R_{\min }, R_{\max }\right)-1}{Z\left(P_{\min }, P_{\max } ; R_{\min }, R_{\max }\right)} .
$$

These updated rates are listed in Table 6. In each case, the results are consistent within the uncertainties but the difference can be used to get a qualitative sense of the systematic uncertainty introduced by the false positive population.

We note that in the above procedure we have not corrected our predictions for the fact that our search has explicitly excluded KOIs that host known Kepler EBs - if any of these excluded systems show fewer than three eclipses and do not present both primary and secondary eclipses, then they should also should perhaps count towards the number of EBs we should have expected to find in this survey. However, as the Kepler EB catalog does not provide information on whether both primary and secondary eclipses are detected, we neglect this correction. We note that this is a conservative decision, in the sense that accounting for the effect of excluded EBs on our predictions would only further decrease the FPP of the planetary signals, as they would be even less likely to be caused by EBs.
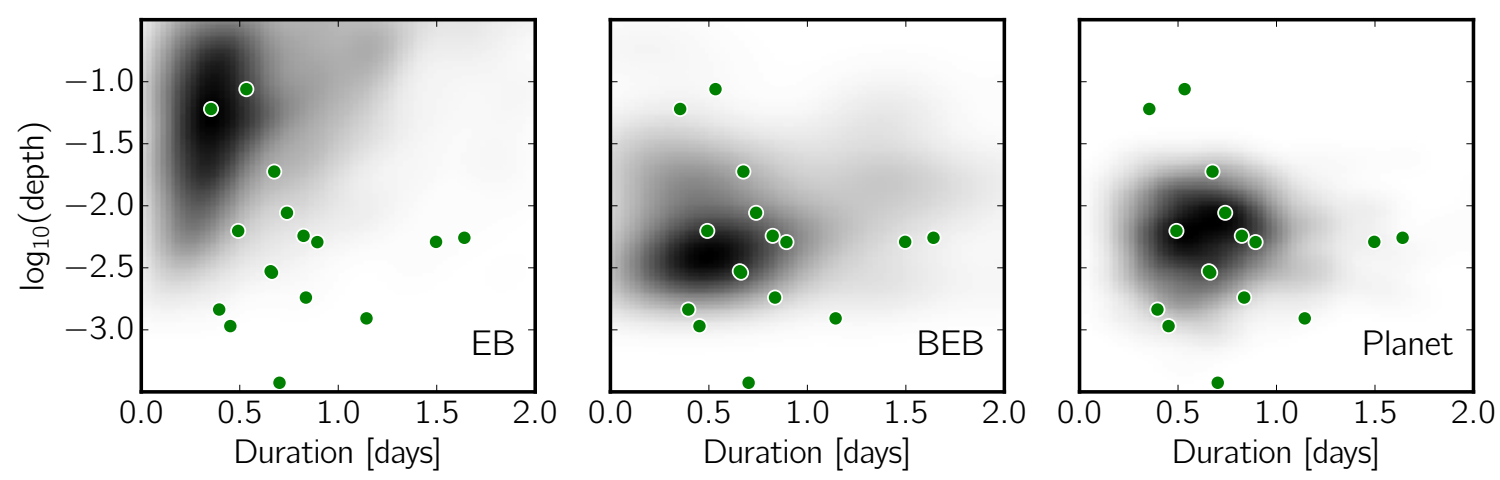

Figure 8. Predicted eclipse shape distributions for the two false positive scenerios and exoplanet transits (grayscale heatmap). In this figure, the relative normalization of the maps are arbitrary but the absolute normalization is discussed in Section 7. The green points show the shape parameters of the long-period exoplanet candidates from Table 2.

\section{COMPARISON WITH THE LITERATURE}

The population of long-period planets has been previously studied using radial velocity, microlensing, and direct imaging surveys. These methods all measure the 
occurrence rate as a function of planet mass instead of radius. Using the transit method, however, we do not directly measure the mass of the planet. Therefore, to compare our results with the literature, we must rely on a mass-radius $(\mathrm{M}-\mathrm{R})$ relationship constructed using exoplanets with both mass and radius measurements (for example Weiss \& Marcy 2014; Wolfgang et al. 2016; Chen \& Kipping 2016) to predict the expected masses of the transiting planets.

Table 7 lists gives constraints on the predicted masses of the exoplanet candidates using the probabilistic M-R relationship from Chen \& Kipping (2016) and taking uncertainties in the planet radius and statistical uncertainties in the $\mathrm{M}-\mathrm{R}$ parameters. We compare the predictions with the predictions from Wolfgang et al. (2016) and find similar values with smaller uncertainties and choose to use the Chen \& Kipping (2016) relationship because it is more conservative in the relevant range of parameter space.

A detailed discussion of the systematic effects introduced by the use of a M-R relationship is beyond the scope of this paper but it is worth noting that all published relationships are based on exoplanets much closer to their host star than any of the candidates discussed here. This effect would cause the masses of these cool planets to be systematically underestimated.

Using the same $\mathrm{M}-\mathrm{R}$ relationship, we also compute the completeness of our transit search as a function of planet mass and orbital semi-major axis. This function is plotted in Figure 9 with the same color scheme as Figure 6. These injections and the predicted masses and measured semi-major axes of the candidates can then be used to estimate the occurrence rate in mass-semi-major axis units using the method from Section 6. One small change to Equation (14) is necessary to account for the fact that the injections were not made uniformly in $\ln M$ and $\ln a$. We numerically estimate the prior distribution in mass and semi-major axis from which the injections were drawn $\tilde{p}(\ln a, \ln M)$ and Equation (14) becomes

$$
Z\left(a_{\min }, a_{\max } ; M_{\min }, M_{\max }\right) \approx \frac{K V}{J} \sum_{j=1}^{J} \frac{Q_{k}\left(w^{(j)}\right)}{\tilde{p}\left(\ln a^{(j)}, \ln M^{(j)}\right)} .
$$

Using this result, we find that the mean occurrence rate density in the range $0.01 M_{\mathrm{J}} \leq$ $M<20 M_{\mathrm{J}}$ and $1.5 \mathrm{au} \leq a<9$ au is

$$
\frac{\mathrm{d}^{2} N}{\mathrm{~d} \ln M \mathrm{~d} \ln a}=0.068 \pm 0.019
$$

where $N$ is the expected number of planets per $\mathrm{G} / \mathrm{K}$ dwarf. This result and the equivalent result as a function of planet mass and orbital period are listed in Table 8 ,

The uncertainty in Equation (19) and Table 8 does not take into account the uncertainties in the mass estimates or any systematic noise in the mass-radius relationship. Therefore, these specific results should be taken with the appropriate grain of salt but predictions in these parameter spaces ease comparison with occurrence rates measured computed using different methods. 
Clanton \& Gaudi (2016) studied the occurrence rate of long-period giant planets orbiting $\mathrm{M}$ dwarfs by combining results from radial velocity, microlensing, and direct imaging surveys. In the period and mass range $10^{3}-10^{4}$ days and $10-10^{4} M_{\oplus}$, they find a mean occurrence rate density of

$$
\frac{\mathrm{d}^{2} N}{\mathrm{~d} \ln M \mathrm{~d} \ln P}=0.023
$$

per $\mathrm{M}$ dwarf with large uncertainty. This result is slightly lower than our estimated rate for a similar range of masses and periods but around $\mathrm{G} / \mathrm{K}$ dwarfs. This difference is consistent with previous observational and theoretical results that cooler stars host fewer long-period giant planets (for example Laughlin et al. 2004; Cumming et al. 2008; Clanton \& Gaudi 2016).

Recently, Bryan et al. (2016) studied the frequency of long-period giant planets in systems with inner hot Jupiters based on long-baseline radial velocity monitoring of these systems (Knutson et al. 2014). In this sample, the computed occurrence rate of long-period giant planets was found to be

$$
\frac{\mathrm{d}^{2} N}{\mathrm{~d} \ln M \mathrm{~d} \ln a}=0.125 \pm 0.012
$$

in the range $1-20 M_{\mathrm{J}}$ and $5-20 \mathrm{au}$. This result is about a factor of two larger than our estimate (Equation 19) once again suggesting that cold Jupiters might preferentially occur in systems with inner planets - or that the presence of cold Jupiters encourages the formation of hot Jupiters.

A recent review of the occurrence rate estimates based on direct imaging surveys Bowler 2016) reports the upper limit on the occurrence rate of giant planets orbiting $\mathrm{F} / \mathrm{G} / \mathrm{K}$ dwarfs as $<6.8 \%$ in the range $5-13 M_{\mathrm{J}}$ and $10-100 \mathrm{au}$. Converted to a rate density, this gives

$$
\frac{\mathrm{d}^{2} N}{\mathrm{~d} \ln M \mathrm{~d} \ln a}<0.03
$$

This value is lower than the value computed using our sample in Table 8 but this is consistent with the fact that direct imaging is sensitive to the potentially less common large planets at wider separations than detections with the transit method.

As a final comparison, we repeated the analysis of Burke et al. (2015) and fit a double power-law occurrence rate to the short-period Kepler planet candidates 7 and extrapolated to the center of the two bins where we computed the occurrence rate. At a period of 7 years and a radius of $0.2 R_{\mathrm{J}}$, the extrapolated occurrence rate density is $0.73 \pm 0.28$ and at a radius of $0.6 R_{\mathrm{J}}$, the extrapolated rate density is $0.15 \pm 0.05$. These extrapolated values are qualitatively consistent with the rates listed in Table 6 but we note that extrapolations and their statistical uncertainties should not be taken too seriously.

\footnotetext{
7 The analysis was adapted from publicly available code that was demonstrated to reproduce the same results as Burke et al. (2015) by Foreman-Mackey (2015).
} 


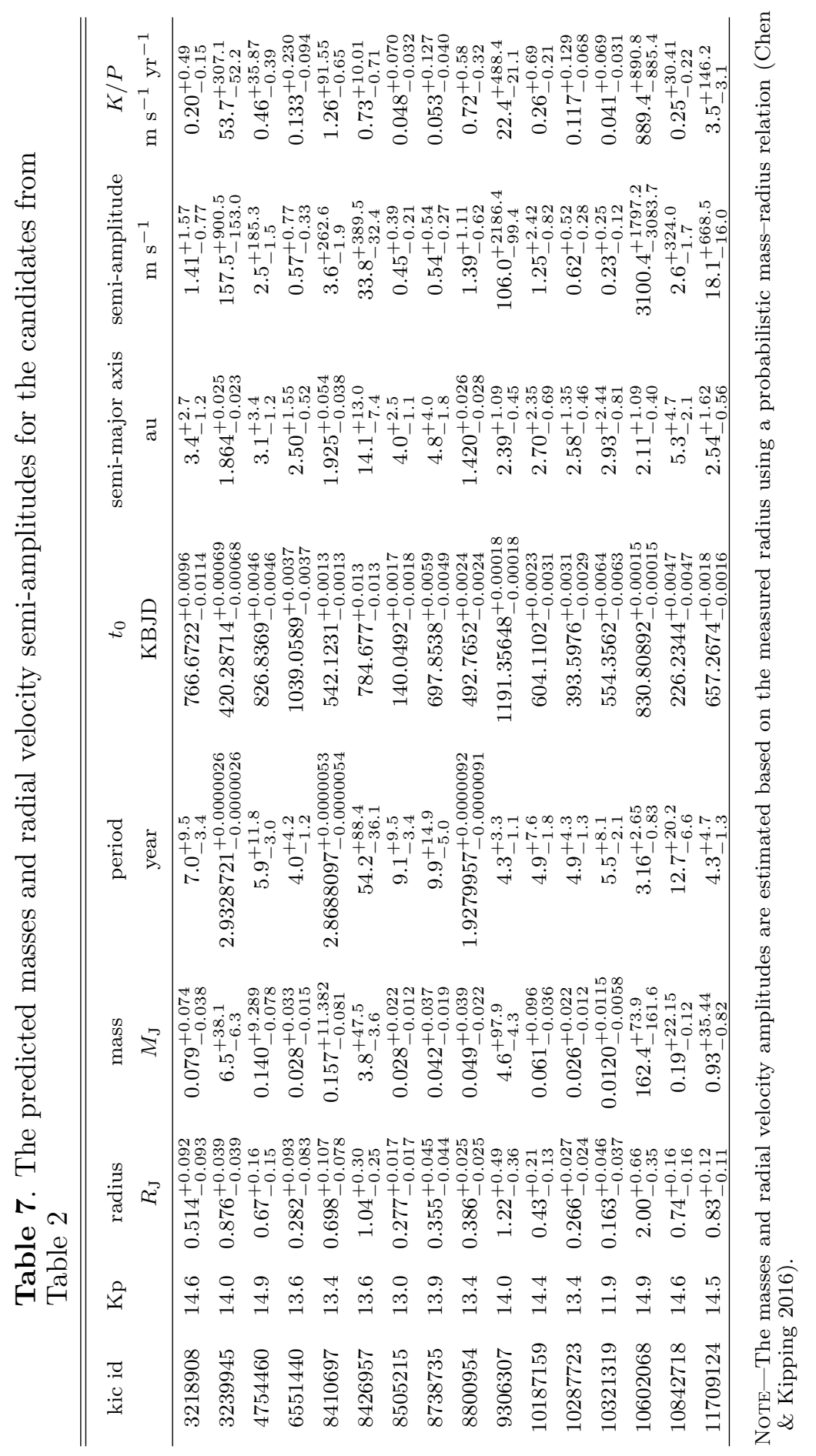


Table 8. The occurrence rate computed in mass units

\begin{tabular}{ccc}
\hline \hline volume & rate density $^{\mathrm{a}}$ & integrated rate \\
\hline $2 \mathrm{yr}<P<25 \mathrm{yr} ; 0.01 M_{\mathrm{J}} \leq M<20 M_{\mathrm{J}}$ & $0.046 \pm 0.013$ & $0.882 \pm 0.245$ \\
$1.5 \mathrm{au}<a<9 \mathrm{au} ; 0.01 M_{\mathrm{J}} \leq M<20 M_{\mathrm{J}}$ & $0.068 \pm 0.019$ & $0.925 \pm 0.257$ \\
\hline$a_{\text {The rate density is measured in natural logarithmic units; see Equation } \sqrt{19}}$. \\
NoTE-These values are computed assuming that the occurrence rate is flat \\
in the logarithmic parameters.
\end{tabular}

\section{PROSPECTS FOR FOLLOW-UP}

A real concern about the detection of exoplanets from a single transit is that followup and confirmation is difficult. Since the period of the orbit is poorly constrained and transits are sparse, any prediction of a subsequent transit time will be too uncertain to schedule targeted photometric follow-up (Beichman et al. 2016; Dalba \& Muirhead 2016). Instead, follow-up using radial velocity and astrometry are more promising. For both radial velocity and astrometry, there is information about the orbiting planets in measurements made at all times - not just during transit. This allows observations to be scheduled without a well constrained orbital period. Furthermore, follow-up of any of these candidates using radial velocity or astrometry would provide a measurement of the density of a planet that would be valuable for the study of planetary compositions.

Table 7 lists the posterior predictions for the semi-amplitude $K$ of the radial velocity signal produced by each candidate using the mass predictions from the previous section. Since the orbital periods are long, we also include a simple prediction for the slope of the radial velocity trend induced by this planet by taking the ratio of the semiamplitude and the orbital period. Any radial velocity follow-up of the candidates presented here would be an ambitious undertaking because the stars are relatively faint and, in most cases, the radial velocity trends are small. Some candidates should, however, be within the reach of current state-of-the-art facilities.

In principle the Gaia Mission will be very sensitive to the astrometric wobble produced by a long-period exoplanet (Perryman et al. 2014). To leading order, the astrometric signal strength is proportional to the semi-major axis of the stellar (primary) reflex motion in angular units. That is, detectability is related to the angle $\alpha$ given by

$$
\alpha=\frac{a}{D} \frac{M_{\mathrm{p}}}{M_{\mathrm{s}}}
$$

where $a$ is the semi-major axis, $D$ is the distance from the observer to the primary, and $M_{\mathrm{p}} / M_{\mathrm{s}}$ is the planet-to-star mass ratio.

The single-visit precision of Gaia will vary with magnitude but is expected to be on the order of $40 \mu$ as at these magnitudes. In detail the confidence with which an exoplanet can be detected or measured in the final Gaia data depends on this 


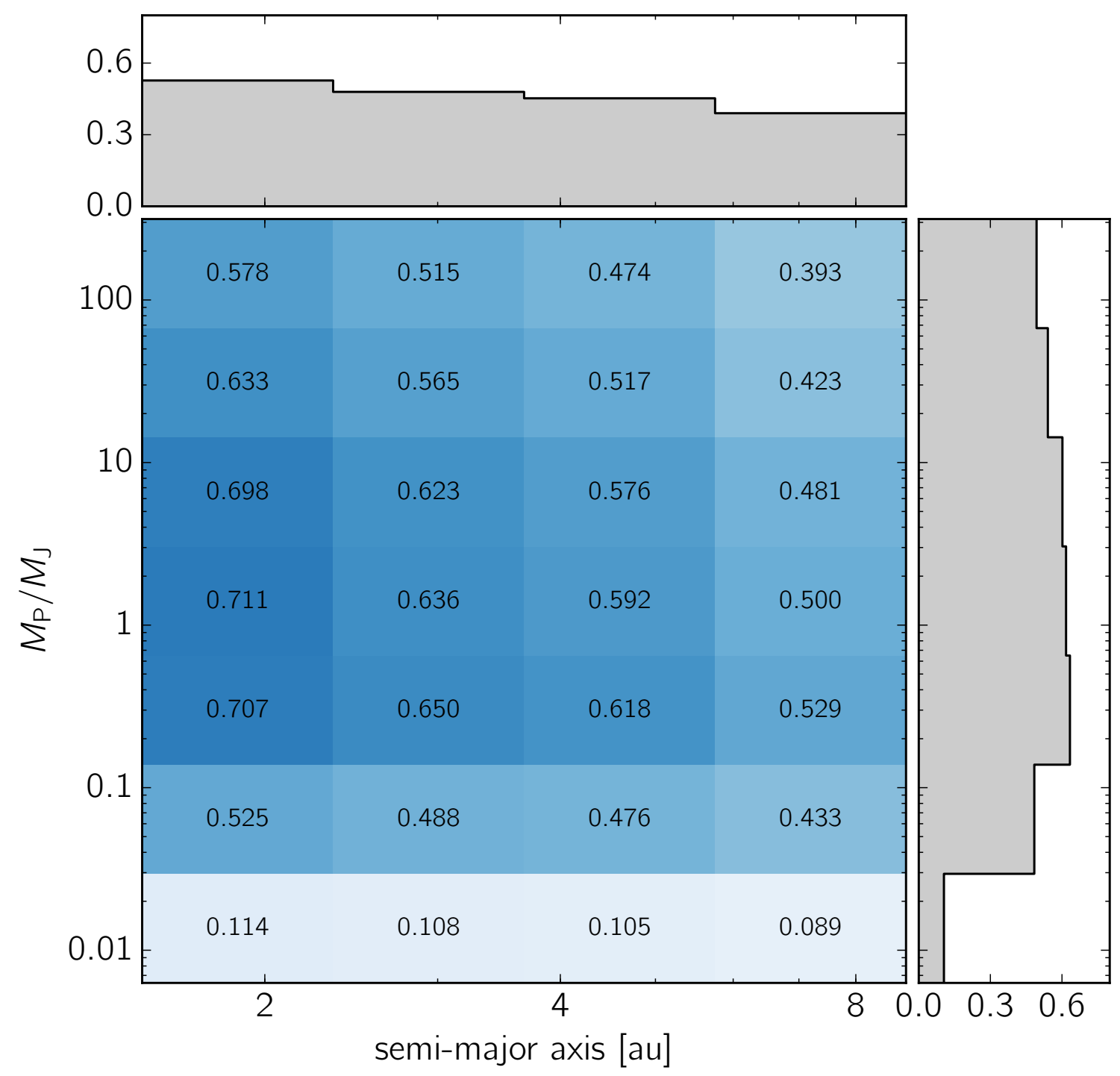

Figure 9. The same as Figure 6 converted into the planet mass and semi-major axis plane. Since the completeness function depends on the planet's radius and not its mass, a probabilistic mass-radius relationship (Chen \& Kipping 2016) was used to convert radius to mass. 
precision, the number of crossings of the star through the Gaia field-of-view, and details of how the projected orbit is sampled by the time history of the focal-plane crossings. However, it is not expected that Gaia can detect or precisely measure exoplanet-induced astrometric wobbles that are much smaller in amplitude than the single-visit precision (Perryman et al. 2014).

The primary stars in the Kepler Field are typically at distances of $\sim 500 \mathrm{pc}$, and typical mass ratios are in the $10^{-4}$ range. We therefore expect astrometric amplitudes in the 0.3 to $3 \mu$ as range. These planets will not be detectable or measurable in the Gaia data under any circumstances, but it may be possible to identify which candidates are false-alarms caused by eclipsing binaries. However, similar planets around closer stars will be detectable with Gaia. This means that there will be a comparable exoplanet occurrence rate measurement from the Gaia data. It also means that many of the discoveries of the K2 and TESS missions could be followed up and precisely measured by the Gaia Mission.

\section{SUMMARY}

We have developed a fully automated method to search for the transits of longperiod exoplanets with only one or two observable transits in the Kepler archival light curves. This method uses probabilistic model comparison to veto non-transit signals. Applying this method to the brightest 39,036 G/K dwarfs in the Kepler target list, we discover 16 systems with likely astrophysical transits and eclipses. We fit the light curve for each candidate with a physical generative model and informative priors on eccentricity and stellar density to estimate the planet's orbital period. The constraint on the period is also informed by the simplifying assumption that no other transit could occur during the baseline of Kepler observations of the target. Simulations of the false positive population - lone primary or secondary eclipses of binary systems or background eclipsing binaries - suggest that 13 of these candidates have high probability of being planetary in nature.

We measure the empirical detection efficiency function of our search procedure by injecting simulated transit signals into the target light curves and measuring the recovery rate. By combining the measured detection efficiency and the catalog of exoplanet candidates, we estimate the integrated occurrence rate of exoplanets with orbital periods in the range $2-25$ years and radii in the range $0.1-1 R_{\mathrm{J}}$ to be $2.00 \pm 0.72$ planets per $\mathrm{G} / \mathrm{K}$ dwarf. This result is qualitatively consistent with estimates of the occurrence rate of long-period giant planets based on data from radial velocity and direct imaging surveys. The occurrence rate measured here - for Sun-like hosts - is higher than microlensing results for generally lower mass stars (Gaudi 2012; Clanton \& Gaudi 2014, 2016) but this discrepancy is consistent with predictions from the core-accretion model (Laughlin et al. 2004).

Using a probabilistic mass-radius relationship, we predict the masses of our candidates and report predictions for the radial velocity semi-amplitudes. Unfortunately, 
since the target stars are faint and the amplitudes are small, these targets are unlikely to be accessible with even the current state-of-the-art high-precision instruments. We also discuss the potential for astrometric follow-up using the forthcoming data from the Gaia Mission with similarly discouraging results.

Any detailed analysis of individual systems detected with only a single transit requires follow-up observations to convincingly rule out false positive scenarios and to better characterize the stellar host parameters (with, for example, parallax measurements from Gaia). The conclusions of this work - and all other occurrence rate results based on Kepler data - are conditioned on the assumption that the stellar characterization of the target sample is systematic and un-biased. The main population-level results should be fairly insensitive systematic issues with the sample but a rigorous analysis of these effects will be required to come to more detailed conclusions about this population of long-period transiting planets.

Our method of transit discovery is especially relevant for future photometric surveys like K2, TESS, and PLATO where the survey baseline is shorter than Kepler. The transits of planets with orbital periods longer than the observation baselines will be plentiful in these forthcoming data sets and this method can, in principle, be trivially generalized to discover these planets, prioritize follow-up, and study their population.

All of the code used in this project is available from https://github.com/dfm/ peerless under the MIT open-source software license. This code (plus some dependencies) can be run to re-generate all of the figures and results in this paper; this version of the paper was generated with git commit a485057 (2016-09-30). The parameter estimation results represented as MCMC samplings and the injection results are available for download from Zenodo at http://dx.doi.org/10.5281/zenodo.58273.

It is a pleasure to thank Jeff Coughlin, So Hattori, Heather Knutson, Phil Muirhead, Darin Ragozzine, Hans-Walter Rix, Dun Wang, and Angie Wolfgang for helpful discussions and contributions. We thank the anonymous referee for comments that improved the presentation and clarity of this manuscript.

T.D.M. was supported by the National Aeronautics and Space Administration, under the Kepler participating scientist program (grant NNX14AE11G), and is grateful for the hospitality of both the Institute for Advanced Study and Carnegie Observatories that helped support this work. D.W.H. was partially supported by the National Science Foundation (grant IIS-1124794), the National Aeronautics and Space Administration (grant NNX12AI50G), and the Moore-Sloan Data Science Environment at NYU. E.A. acknowledges support from NASA grants NNX13AF20G, NNX13AF62G, and NASA Astrobiology Institutes Virtual Planetary Laboratory, supported by NASA under cooperative agreement NNH05ZDA001C. 
This research made use of the NASA Astrophysics Data System and the NASA Exoplanet Archive. The Exoplanet Archive is operated by the California Institute of Technology, under contract with NASA under the Exoplanet Exploration Program.

This paper includes data collected by the Kepler mission. Funding for the Kepler mission is provided by the NASA Science Mission directorate. We are grateful to the entire Kepler team, past and present. Their tireless efforts were all essential to the tremendous success of the mission and the successes of $K 2$, present and future.

These data were obtained from the Mikulski Archive for Space Telescopes (MAST). STScI is operated by the Association of Universities for Research in Astronomy, Inc., under NASA contract NAS5-26555. Support for MAST is provided by the NASA Office of Space Science via grant NNX13AC07G and by other grants and contracts.

Computing resources were provided by High Performance Computing at New York University.

Facility: Kepler

Software: batman (Kreidberg 2015), ceres (Agarwal et al. 2016), corner.py (Foreman-Mackey 2016), emcee (Foreman-Mackey et al. 2013), exosyspop (Morton \& Foreman-Mackey 2016), george (Ambikasaran et al.|2016), isochrones (Morton 2015a), matplotlib (Hunter et al. 2007), numpy (Van Der Walt et al.|2011), scipy (Jones et al. 2001), transit (Foreman-Mackey \& Morton 2016), vespa (Morton 2015b). 


\section{APPENDIX}

\section{A. DETAILS OF THE LIGHT CURVE MODELS}

In Section 2.2, the five light curve models were listed. In this section, we give the mathematical details of each model and list the parameters that are fit. Each model - except the transit model - can be easily differentiated with respect to its parameters. As discussed in the following section, this feature is crucial for efficient and robust likelihood maximization.

- The box model is given by

$$
m_{\mathrm{box}}(t)=\left\{\begin{array}{l}
a, \text { if } t \leq t_{\min } \\
b, \text { if } t_{\min }<t \leq t_{\max } \\
c, \text { if } t_{\max }<t
\end{array}\right.
$$

where $a, b$, and $c$ are free parameters, and $t_{\min }$ and $t_{\max }$ are fixed. In practice, we include two different box models where $t_{\min }$ and $t_{\max }$ are set using different heuristics. The first box has the bounds set to match the ingress and egress of the best fit transit. The second box is chosen based on the largest change points in the light curve.

- The step model is given by

$$
m_{\text {step }}(t)=\left\{\begin{array}{l}
m_{1}+h_{1} \exp \left(\left[t-t_{0}\right] / w_{1}\right), \text { if } t<t_{0} \\
m_{2}+h_{2} \exp \left(\left[t_{0}-t\right] / w_{2}\right), \text { if } t_{0} \leq t
\end{array}\right.
$$

where all of the parameters - including $t_{0}$ - are included in the fit. To ensure that the widths $w_{1}$ and $w_{2}$ remain positive, we fit for $\log w_{1}$ and $\log w_{2}$.

- For the outlier model, we iterate through all cadences $t_{n}$ within 0.3 days of the candidate transit time and evaluate the model as

$$
m_{\text {outlier }}(t)=\left\{\begin{array}{lr}
f\left(t_{0}\right), & \text { if } t=t_{0} \\
\operatorname{median}\left[f\left(t \neq t_{0}\right)\right], & \text { if } t \neq t_{0}
\end{array}\right.
$$

where $f(t)$ is the observed time series. With this model, no non-linear optimization is required and the final value of $t_{0}$ is the one with the maximum likelihood in this grid search.

- The variability model only has one parameter, the flux $m_{0}$ and $m_{\text {variability }}(t)=m_{0}$ at all times. The variability is captured by the Gaussian Process residual model.

- Finally, the transit model is an exposure time integrated, limb darkened light curve (Mandel \& Agol 2002; Kipping 2010) parameterized by the radius ratio between the planet and star, the transit duration, the transit time, the impact parameter, 
and two quadratic limb darkening coefficients (Kipping 2013a). Analytically computing the gradient of a simple transit model is possible (Pál 2008) but it becomes substantially more tedious as the model becomes more realistic. Therefore, we instead use a compile-time automatic differentiation library ${ }^{8}$ Agarwal et al. 2016) to efficiently compute first derivatives of the full transit model with respect to the orbital and physical parameters to machine precision.

\section{B. GAUSSIAN PROCESS REGRESSION}

Gaussian Processes (GPs) are a class of non-parametric, stochastic models that have been demonstrated to be good effective models for the variability in Kepler light curves. A simple GP model can be used to capture residual non-transit variability in light curves. In this paper, we use a GP model for two steps: light curve-level transit shape vetting and parameter estimation. A full discussion of GPs is beyond the scope of this paper, so we will only summarize the most relevant points here and direct an interested reader to Rasmussen \& Williams (2006) for more details.

A GP model is specified by the following likelihood function

$$
\mathcal{L}=\ln p(\boldsymbol{y} \mid \boldsymbol{\theta}, \boldsymbol{\alpha})=-\frac{1}{2} \boldsymbol{r}(\boldsymbol{\theta})^{\mathrm{T}} K(\boldsymbol{\alpha})^{-1} \boldsymbol{r}(\boldsymbol{\theta})-\frac{1}{2} \log \operatorname{det} K(\boldsymbol{\alpha})-\frac{N}{2} \log 2 \pi
$$

where $\boldsymbol{y}$ is a list of measurements in a scalar time series - in this case, fluxes - measured at the times $\boldsymbol{t}$, and

$$
\boldsymbol{r}(\boldsymbol{\theta})=\boldsymbol{y}-m(\boldsymbol{t} ; \boldsymbol{\theta})
$$

is the vector of residuals away from the mean model $m(\boldsymbol{t} ; \boldsymbol{\theta})$. For the purposes of this paper, we model the covariance matrix $K(\boldsymbol{\alpha})$ using the Matérn-3/2 kernel. Under this model, the elements of $K(\boldsymbol{\alpha})$ are given by

$$
[K(\boldsymbol{\alpha})]_{i j}=\sigma_{i}^{2} \delta_{i j}+\alpha^{2}\left[1+\frac{\left|t_{i}-t_{j}\right|}{\sqrt{3} \tau}\right] \exp \left(-\frac{\left|t_{i}-t_{j}\right|}{\sqrt{3} \tau}\right)
$$

where $\sigma_{i}$ is the reported uncertainty on the $i$-th measurement in the time series and $\delta_{i j}$ is the Kronecker delta.

This covariance function (Equation $\mathrm{B6}$ ) is specified by an amplitude $\alpha$ and a time scale $\tau$ and we will simultaneously fit for these hyperparameters $\boldsymbol{\alpha}=(\alpha, \tau)$ and the parameters of the mean model $\boldsymbol{\theta}$. To efficiently find the parameter set that maximizes Equation (B4) using a non-linear optimization routine ${ }^{9}$, it is useful to be able to compute the gradient of Equation $(\sqrt{\mathrm{B} 4})$ with respect to the parameters $\boldsymbol{\theta}$ and $\boldsymbol{\alpha}$. These gradients are given by

$$
\frac{\mathrm{d} \ln p(\boldsymbol{y} \mid \boldsymbol{\theta}, \boldsymbol{\alpha})}{\mathrm{d} \boldsymbol{\theta}}=\frac{\mathrm{d} m(\boldsymbol{t} ; \boldsymbol{\theta})^{\mathrm{T}}}{\mathrm{d} \boldsymbol{\theta}} K(\boldsymbol{\alpha})^{-1} \boldsymbol{r}(\boldsymbol{\theta})
$$

8 More specifically, we use the Jet object from the BSD-licensed Ceres Solver http:// ceres-solver.org

${ }^{9}$ We use the L-BFGS-B method as implemented in SciPy http://docs.scipy.org/doc/scipy/ reference/generated/scipy.optimize.minimize.html. 
and

$$
\frac{\mathrm{d} \ln p(\boldsymbol{y} \mid \boldsymbol{\theta}, \boldsymbol{\alpha})}{\mathrm{d} \boldsymbol{\alpha}}=\frac{1}{2} \operatorname{Tr}\left(\left[\boldsymbol{\phi} \boldsymbol{\phi}^{\mathrm{T}}-K(\boldsymbol{\alpha})^{-1}\right] \frac{\mathrm{d} K(\boldsymbol{\alpha})}{\mathrm{d} \boldsymbol{\alpha}}\right)
$$

where

$$
\boldsymbol{\phi}=K(\boldsymbol{\alpha})^{-1} \boldsymbol{r}(\boldsymbol{\theta})
$$

\section{REFERENCES}

Agarwal, S., Mierle, K., \& et al. 2016, Ceres Solver, http://ceres-solver.org, ,

Ambikasaran, S., Foreman-Mackey, D.,

Greengard, L., Hogg, D., \& O Neil, M. 2016, IEEE

Ballard, S., \& Johnson, J. A. 2016, ApJ, 816, 66

Beichman, C., Livingston, J., Werner, M., et al. 2016, ApJ, 822, 39

Borucki, W. J., Koch, D. G., Basri, G., et al. 2011, ApJ, 736, 19

Bowler, B. P. 2016, ArXiv e-prints, arXiv:1605.02731

Bryan, M. L., Knutson, H. A., Howard, A. W., et al. 2016, ApJ, 821, 89

Bryson, S. T., Jenkins, J. M., Gilliland, R. L., et al. 2013, PASP, 125, 889

Burke, C. J., \& McCullough, P. R. 2014, ApJ, 792,79

Burke, C. J., Bryson, S. T., Mullally, F., et al. 2014, ApJS, 210, 19

Burke, C. J., Christiansen, J. L., Mullally, F., et al. 2015, ApJ, 809, 8

Cassan, A., Kubas, D., Beaulieu, J.-P., et al. 2012, Nature, 481, 167

Chen, J., \& Kipping, D. M. 2016, ArXiv e-prints, arXiv:1603.08614

Christiansen, J. L., Clarke, B. D., Burke, C. J., et al. 2013, ApJS, 207, 35

—. 2015, ApJ, 810, 95

Clanton, C., \& Gaudi, B. S. 2014, ApJ, 791, 91

—. 2016, ApJ, 819, 125

Coughlin, J. L., Mullally, F., Thompson, S. E., et al. 2016, ApJS, 224, 12

Cumming, A., Butler, R. P., Marcy, G. W., et al. 2008, PASP, 120, 531

Dalba, P. A., \& Muirhead, P. S. 2016, ApJL, 826, L7

Dalba, P. A., Muirhead, P. S., Fortney, J. J., et al. 2015, ApJ, 814, 154

Dawson, R. I., \& Johnson, J. A. 2012, ApJ, 756,122

Dressing, C. D., \& Charbonneau, D. 2015, ApJ, 807, 45

Fang, J., \& Margot, J.-L. 2012, ApJ, 761, 92

Fischer, D. A., Schwamb, M. E., Schawinski, K., et al. 2012, MNRAS, 419, 2900
Foreman-Mackey, D. 2015, An experiment in open science: exoplanet population inference, http://dfm.io/posts/exopop/, Zenodo, doi:10.5281/zenodo.57218

-. 2016, The Journal of Open Source Software, 24, doi:10.21105/joss.00024

Foreman-Mackey, D., Hogg, D. W., Lang, D., \& Goodman, J. 2013, PASP, 125, 306

Foreman-Mackey, D., Hogg, D. W., \& Morton, T. D. 2014, ApJ, 795, 64

Foreman-Mackey, D., Montet, B. T., Hogg, D. W., et al. 2015, ApJ, 806, 215

Foreman-Mackey, D., \& Morton, T. 2016, $\mathrm{dfm} /$ transit: v0.3.0, , , doi:10.5281/zenodo.159478

Gaidos, E., \& Mann, A. W. 2012, ApJ, 762, 41

Gaudi, B. S. 2012, ARA\&A, 50, 411

Girardi, L., Groenewegen, M. A. T., Hatziminaoglou, E., \& da Costa, L. 2005, A\&A, 436, 895

Gould, A., Gaudi, B. S., \& Han, C. 2004, ArXiv Astrophysics e-prints, astro-ph/0405217

Gould, A., Dong, S., Gaudi, B. S., et al. 2010, ApJ, 720, 1073

Howard, A. W., Marcy, G. W., Bryson, S. T., et al. 2012, ApJS, 201, 15

Howell, S. B., Sobeck, C., Haas, M., et al. 2014, PASP, 126, 398

Huber, D., Silva Aguirre, V., Matthews, J. M., et al. 2014, ApJS, 211, 2

Hunter, J. D., et al. 2007, Computing in science and engineering, 9, 90

Jones, E., Oliphant, T., Peterson, P., et al. 2001, SciPy: Open source scientific tools for Python, ,

Kipping, D. M. 2010, MNRAS, 408, 1758

-. 2013a, MNRAS, 435, 2152

—. 2013b, MNRAS, 434, L51

Kipping, D. M., Bastien, F. A., Stassun, K. G., et al. 2014a, ApJL, 785, L32

Kipping, D. M., Dunn, W. R., Jasinski, J. M., \& Manthri, V. P. 2012, MNRAS, 421, 1166

Kipping, D. M., Torres, G., Buchhave, L. A., et al. 2014b, ApJ, 795, 25

Kipping, D. M., Torres, G., Henze, C., et al. 2016, ApJ, 820, 112 
Kirk, B., Conroy, K., Prša, A., et al. 2016, AJ, 151,68

Knutson, H. A., Fulton, B. J., Montet, B. T., et al. 2014, ApJ, 785, 126

Kovács, G., Zucker, S., \& Mazeh, T. 2002, A\&A, 391, 369

Kreidberg, L. 2015, PASP, 127, 1161

Laughlin, G., Bodenheimer, P., \& Adams, F. C. 2004, ApJL, 612, L73

Mandel, K., \& Agol, E. 2002, ApJL, 580, L171

Moriarty, J., \& Ballard, S. 2015, ArXiv e-prints, arXiv:1512.03445

Morton, T., \& Foreman-Mackey, D. 2016, timothydmorton/exosyspop: v0.1-alpha, , , doi:10.5281/zenodo.159487

Morton, T. D. 2012, ApJ, 761, 6

—. 2015a, isochrones: Stellar model grid package, Astrophysics Source Code Library, , , ascl:1503.010

—. 2015b, VESPA: False positive probabilities calculator, Astrophysics Source Code Library, , , ascl:1503.011

Morton, T. D., Bryson, S. T., Coughlin, J. L., et al. 2016, ApJ, 822, 86

Morton, T. D., \& Johnson, J. A. 2011, ApJ, 738,170

Mulders, G. D., Pascucci, I., \& Apai, D. 2015, ApJ, 814, 130

Mullally, F., Coughlin, J. L., Thompson, S. E., et al. 2016, PASP, 128, 074502

Osborn, H. P., Armstrong, D. J., Brown, D. J. A., et al. 2016, MNRAS, 457, 2273

Pál, A. 2008, MNRAS, 390, 281

Perryman, M., Hartman, J., Bakos, G. Á., \& Lindegren, L. 2014, ApJ, 797, 14

Petigura, E. A., Howard, A. W., \& Marcy, G. W. 2013, Proceedings of the National Academy of Science, 110, 19273
Rasmussen, C. E., \& Williams, C. K. I. 2006, Gaussian processes for machine learning (MIT Press)

Rauer, H., Catala, C., Aerts, C., et al. 2014, Experimental Astronomy, 38, 249

Ricker, G. R., Winn, J. N., Vanderspek, R., et al. 2015, Journal of Astronomical Telescopes, Instruments, and Systems, 1, 014003

Rogers, L. A. 2015, ApJ, 801, 41

Rowe, J. F., Coughlin, J. L., Antoci, V., et al. 2015, ApJS, 217, 16

Shvartzvald, Y., Maoz, D., Udalski, A., et al. 2016, MNRAS, 457, 4089

Smith, J. C., Stumpe, M. C., Van Cleve, J. E., et al. 2012, PASP, 124, 1000

Stumpe, M. C., Smith, J. C., Van Cleve, J. E., et al. 2012, PASP, 124, 985

Tremaine, S., \& Dong, S. 2012, AJ, 143, 94

Uehara, S., Kawahara, H., Masuda, K., Yamada, S., \& Aizawa, M. 2016, ApJ, 822, 2

Van Der Walt, S., Colbert, S. C., \& Varoquaux, G. 2011, Computing in Science \& Engineering, 13, 22

Wang, J., Fischer, D. A., Barclay, T., et al. 2013, ApJ, 776, 10

-. 2015, ApJ, 815, 127

Weiss, L. M., \& Marcy, G. W. 2014, ApJL, $783, \mathrm{~L} 6$

Winn, J. N. 2010, ArXiv e-prints, arXiv: 1001.2010

Wolfgang, A., Rogers, L. A., \& Ford, E. B. 2016, ApJ, 825, 19

Yee, J. C., \& Gaudi, B. S. 2008, ApJ, 688, 616 